\title{
THE ORIGIN OF PENUMBRAL STRUCTURE IN SUNSPOTS: DOWNWARD PUMPING OF MAGNETIC FLUX
}

\author{
Nigel O. Weiss, ${ }^{1}$ John H. Thomas, ${ }^{2}$ Nicholas H. Brummell, ${ }^{3}$ and Steven M. Tobias ${ }^{4}$ \\ Received 2003 June 5; accepted 2003 September 22
}

\begin{abstract}
This paper offers the first coherent picture of the interactions between convection and magnetic fields that lead to the formation of the complicated filamentary structure of a sunspot penumbra. Recent observations have revealed the intricate interlocking-comb structure of the penumbral magnetic field. Some field lines, with associated Evershed outflows, plunge below the solar surface near the edge of the spot. We claim that these field lines are pumped downward by small-scale granular convection outside the sunspot. This mechanism is demonstrated in numerical experiments. Magnetic pumping is a key new ingredient that links several theoretical ideas about penumbral structure and dynamics; it explains not only the abrupt appearance of a penumbra as a pore increases in size but also the behavior of moving magnetic features outside a spot.
\end{abstract}

Subject headings: MHD — Sun: magnetic fields — Sun: photosphere — sunspots

On-line material: color figures

\section{INTRODUCTION}

Sunspots have been observed through telescopes for almost 400 years, but they still raise fundamental questions that are hard to answer. Galileo and Scheiner were already aware of the distinction between the dark umbra and the annular penumbra that surrounds it. Two hundred years later, with the development of much larger telescopes, it was recognized that the penumbra is composed of alternating bright and dark filaments. Since then, the attainable resolution has progressively improved, culminating in the remarkable images that are now being obtained with the Swedish $1 \mathrm{~m}$ Solar Telescope (Scharmer et al. 2002). Figure 1 shows part of the penumbra of a spot, with the individual filaments revealed in unprecedented detail. Hale showed, a century ago, that sunspots resulted from the presence of a strong magnetic field, but the intricate threedimensional geometry of the field in penumbral filaments has only been revealed in the last 10 years (Thomas \& Weiss 1992; Martínez Pillet 1997; del Toro Iniesta 2001; Solanki 2003). This interlocking structure is surprisingly complicated, and its origin has remained a puzzle. Here we argue that it is formed as a consequence of magnetic pumping by the turbulent convection that shows up as solar granulation. Magnetic pumping provides a key new ingredient that links several theoretical ideas about the formation and structure of the penumbra into a coherent picture.

It has long been known that the azimuthally averaged magnetic field in a sunspot is inclined to the vertical at an angle that increases with increasing radius, reaching a value of about $70^{\circ}$ at the edge of the spot, as sketched in Figure $2 a$ (Bray \& Loughhead 1964; Skumanich 1992; Stix 2002). Thus, it was natural to suppose that bright and dark filaments were alternate phases of some convectively driven interchange. Such a picture

\footnotetext{
${ }^{1}$ Department of Applied Mathematics and Theoretical Physics, University of Cambridge, Cambridge CB3 0WA, UK; n.o.weiss@damtp.cam.ac.uk.

2 Department of Physics and Astronomy and Department of Mechanical Engineering, University of Rochester, Rochester, NY 14627-0171; thomas@ me.rochester.edu.

3 JILA and Department of Astrophysical and Planetary Sciences, University of Colorado at Boulder, Boulder, CO 80309-0440; brummell@solarz.colorado .edu.

${ }^{4}$ Department of Applied Mathematics, University of Leeds, Leeds LS2 9JT, UK; smt@maths.leeds.ac.uk.
}

would be appropriate for a shallow penumbra with an almost horizontal field (Danielson 1961), but it was soon realized that this raised a contradiction. On the one hand, much of the spot's magnetic flux emerges through the penumbra, and so the field must have a substantial vertical component; on the other, the horizontal Evershed outflow must be parallel to the magnetic field, which therefore also has to be horizontal. This suggests that there should be some systematic difference between the inclinations of magnetic fields in bright and dark filaments, as first proposed by Beckers \& Schröter (1969). High-resolution observations (Degenhardt \& Wiehr 1991; Title et al. 1993; Lites et al. 1993; Solanki \& Montavon 1993) have revealed that this is indeed so and that the two components differ in inclination by $30^{\circ}-40^{\circ}$. At the umbral-penumbral boundary, the fields in the bright and dark filaments are inclined at about $30^{\circ}$ and $60^{\circ}$, respectively, to the vertical, while at the outer edge of the penumbra the field in the dark filaments is almost horizontal, as sketched in Figure $2 b$. This interlocking-comb (or "uncombed") geometry is confirmed by velocity measurements (Title et al. 1993; Solanki, Montavon, \& Livingston 1994). Furthermore, while the fields from dark filaments typically emerge to form a shallow canopy that hugs the solar surface (or even dive beneath it), loops that emerge from bright filaments extend over great distances across the Sun, as shown by both X-ray observations (Sams, Golub, \& Weiss 1992) and the striking EUV images from TRACE (Winebarger, DeLuca, \& Golub 2001; Winebarger et al. 2002). Figure 3 shows bright loops that connect the penumbrae of a well-spaced pair of sunspots. There is no way that fields in these long loops can interchange with the horizontal fields that carry the Evershed flow. Thus, the bright and dark filaments must remain distinct.

We are therefore led to a new picture of a two-component penumbra, which differs fundamentally from the old picture of interchanges in an almost axisymmetric system. This raises two important questions. First, how is this extraordinary structure maintained, and second, what is the origin of this geometry? We propose here that the filamentary structure is caused by downward pumping of magnetic flux by turbulent convection in the granulation outside the sunspot, following an initial convectively driven instability. As a preliminary step, we present numerical simulations that illustrate one aspect of this process. 


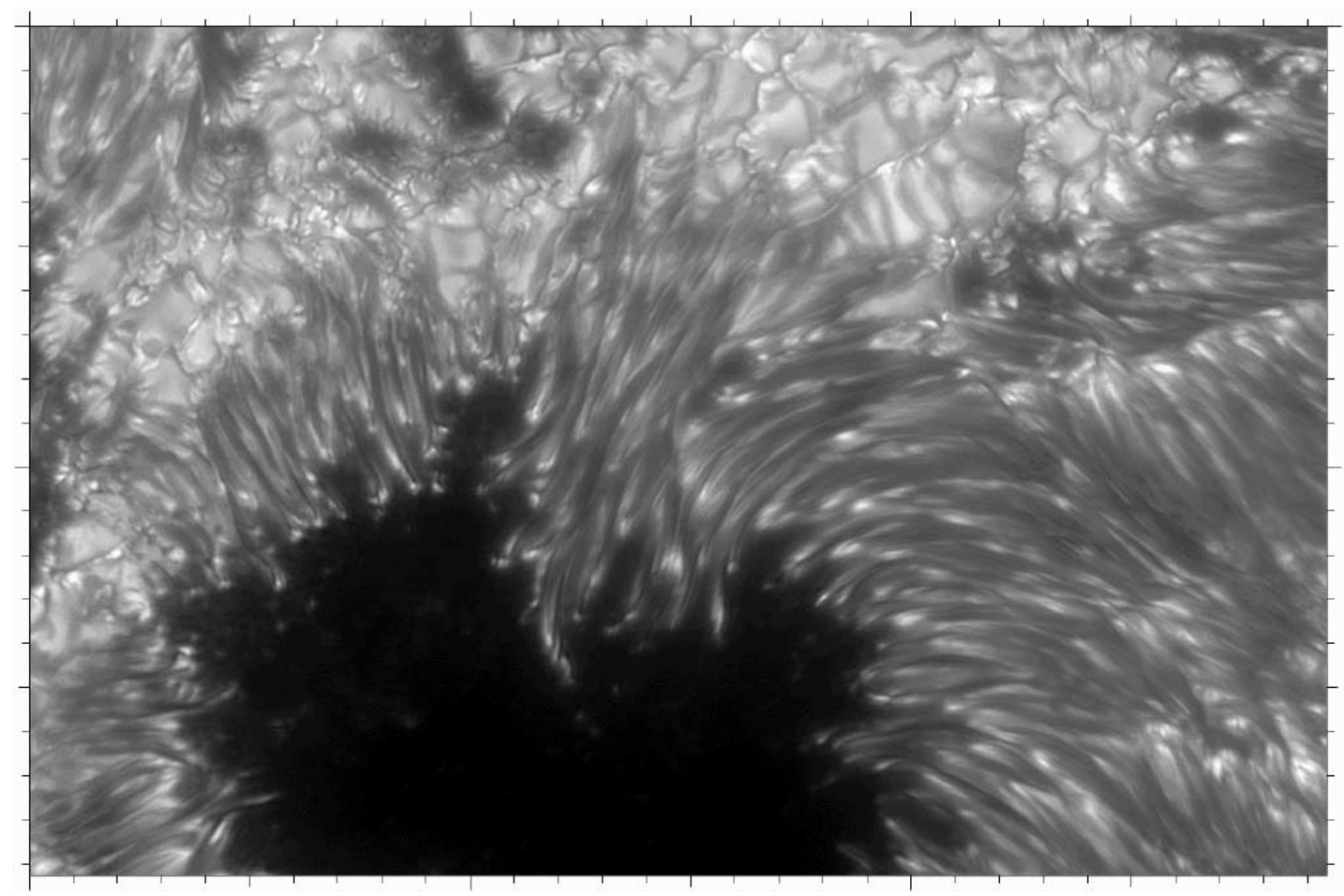

FIG. 1. - G-band image of a portion of a sunspot penumbra, taken with the Swedish $1 \mathrm{~m}$ solar telescope (courtesy of the Royal Swedish Academy of Sciences)

In the next section we describe our physical picture and use some recent observations to argue that flux pumping has to be important. This process is discussed in $\S 3$, and $\S 4$ presents some new model calculations that demonstrate flux pumping in idealized configurations. In $\S 5$ we consider the implications of the two-component model for penumbral convection. Next, we discuss the Evershed flow in $\S 6$, followed in $\S 7$ by an account of moving magnetic features in the moat cell that surrounds a spot. Then, in $\S 8$ we attempt to explain how the filamentary penumbra (which distinguishes sunspots from pores) is formed. The final section provides a summary of the paper. Our main results were announced by Thomas et al. (2002b) and have also been summarized in a conference proceedings (Thomas et al. 2002a).

\section{PHYSICAL PICTURE}

The key to understanding the magnetic field configuration in the penumbra is the existence of numerous arched, "returning" magnetic flux tubes that emerge from below the surface in the inner or middle penumbra and dive back down below the surface in the outer penumbra or just outside the penumbra. The existence of these returning flux tubes has gradually been revealed over the last decade by high-resolution observations and more sophisticated inversions of Stokes profiles.

The returning penumbral flux tubes are intimately connected with the photospheric Evershed flow. Because of the relatively high electrical conductivity of the gas, the Evershed outflow is constrained to be along magnetic field lines, and thus measurements of the Evershed velocity indirectly reveal some aspects of the configuration of the penumbral magnetic field. The Evershed flow is largely confined to the dark penumbral filaments (Beckers 1968), in which the magnetic field is more nearly horizontal (Beckers \& Schröter 1969). Some of the nearly horizontal magnetic field extends radially outward beyond the penumbral boundary along a magnetic "canopy," elevated slightly above the surrounding quiet photosphere. A small fraction of the Evershed flow is observed to continue outward along the canopy (Solanki et al. 1994), but we now know that most of the Evershed flow runs along returning flux tubes and plunges back down below the surface at points either just within or just beyond the outer penumbral boundary. This configuration was hinted at in earlier velocity measurements (Börner \& Kneer 1992; Rimmele 1995a). Next came more solid evidence of downflows in the descending parts of arched flux tubes, apparently ending at outer footpoints corresponding to strong magnetic elements (Rimmele 1995b; Stanchfield, Thomas, \& Lites 1997). Then Westendorp Plaza et al. (1997) established this configuration convincingly through careful inversions of the Stokes profiles that determine the magnetic field and flow velocity as functions of optical depth, thus allowing them to trace flow velocity and magnetic field along the arched flux tube. Subsequent observations have confirmed this configuration (Krivtsov et al. 1998; Rüedi, Solanki, \& Keller 1999; Schlichenmaier \& Schmidt 2000).

The returning flux tube configuration of the Evershed flow nicely explains the sudden disappearance of the Evershed effect near the outer edge of the penumbra. Furthermore, this configuration is in good agreement with the siphon flow model of the Evershed flow (e.g., Montesinos \& Thomas 1997), in which a flow along an arched magnetic flux tube is driven by a drop in gas pressure between the two footpoints of the arch (see $\S 6$ ). Indeed, recent observations have even revealed supersonic Evershed flows in the downstream legs of the returning flux tubes (del Toro Iniesta, Bellot Rubio, \& Collados 2001; Hirzberger \& Kneer 2001), as predicted by the siphon flow model.

The well-established existence of the returning penumbral flux tubes raises an important question, the answer to which provides new insight into the structure of a sunspot. The question is, what holds the submerged part of the flux tube 
(a)
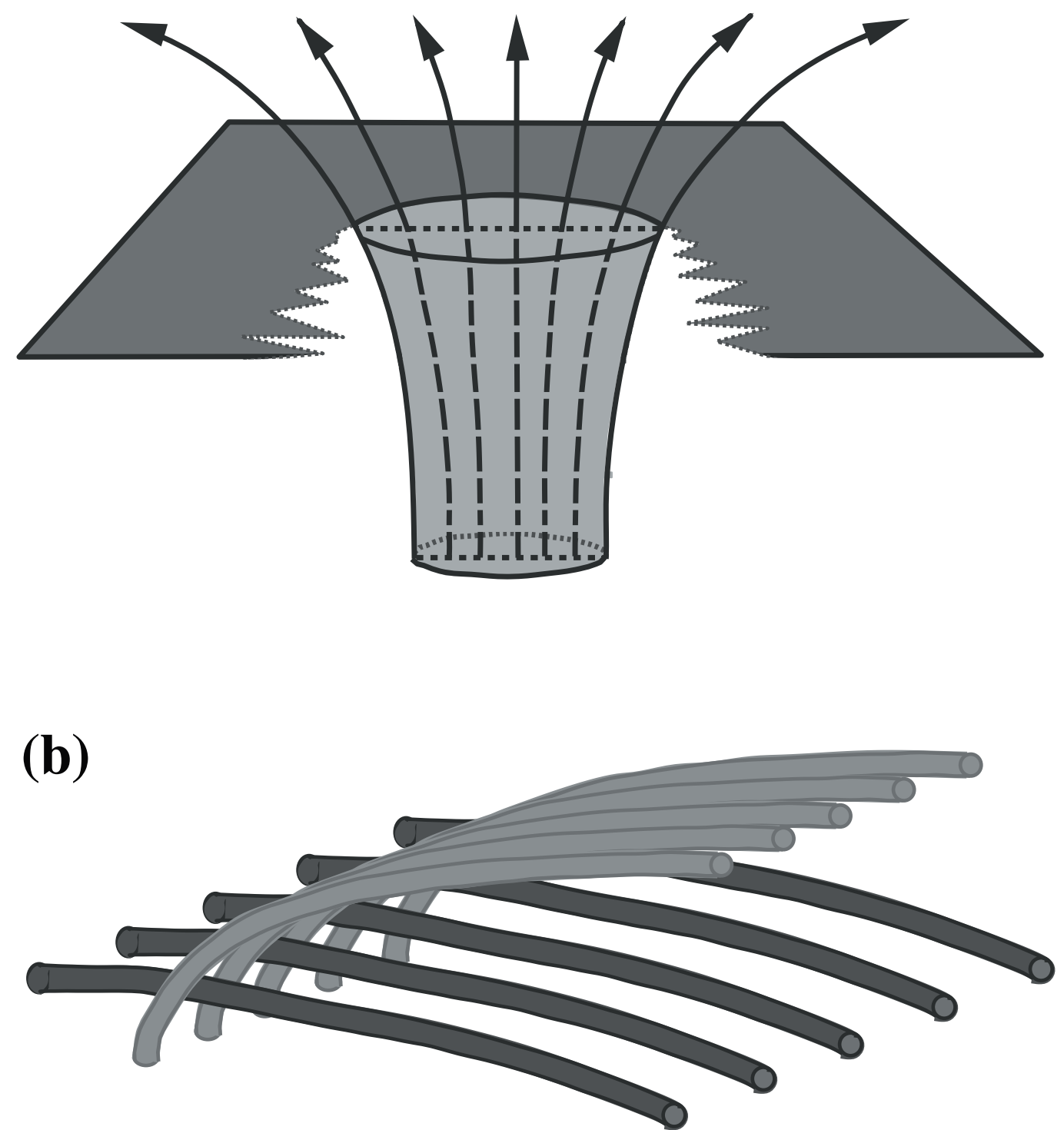

FIG. 2.-Sketches of $(a)$ an axisymmetric magnetic field configuration in a sunspot and $(b)$ the interlocking-comb magnetic field configuration in a sunspot penumbra.

down below the surface, in opposition to the magnetic buoyancy and curvature forces that might be expected to bring the tube quickly up to the surface? We claim that flux tubes are dragged downward as a result of turbulent pumping by the granular convection in the immediate surroundings of the sunspot (the "moat"). We have already demonstrated the effectiveness of this mechanism with idealized numerical simulations (Thomas et al. 2002a, 2002b), and here (in $\S$ 4) we add further support by extending these calculations to a more appropriate parameter regime.

The new ingredient of magnetic flux pumping leads us to the overall picture of a sunspot sketched in Figure 4. The bright radial filaments, where the magnetic field is tilted (at about $40^{\circ}$ to the horizontal in the outer penumbra), alternate with dark filaments in which the field is nearly horizontal. Within the dark filaments, some magnetic flux tubes extend radially outward beyond the penumbra along the elevated magnetic canopy, while other, returning flux tubes dive back below the surface. The submerged parts of the returning flux tubes are held down as a result of turbulent pumping (large red vertical arrows) by the small-scale granular convection (small black squiggly arrows) in the immediate surroundings of the sunspot. Superimposed on the granular convection is the slow radial outflow associated with a long-lived annular supergranule (the moat cell; large curved red arrow).

\section{FLUX PUMPING}

In this section we first explain the various mechanisms that lead to the expulsion of magnetic flux from laminar and turbulent flows, emphasizing the role of sinking plumes in pumping fields preferentially downward. Then we discuss the relevance of flux pumping in the Sun, both at the base of the convection zone and at the photosphere.

\subsection{Flux Expulsion and Flux Pumping}

Flux expulsion is a common feature of cellular convection in a highly conducting fluid (Weiss 2003). The simplest examples 


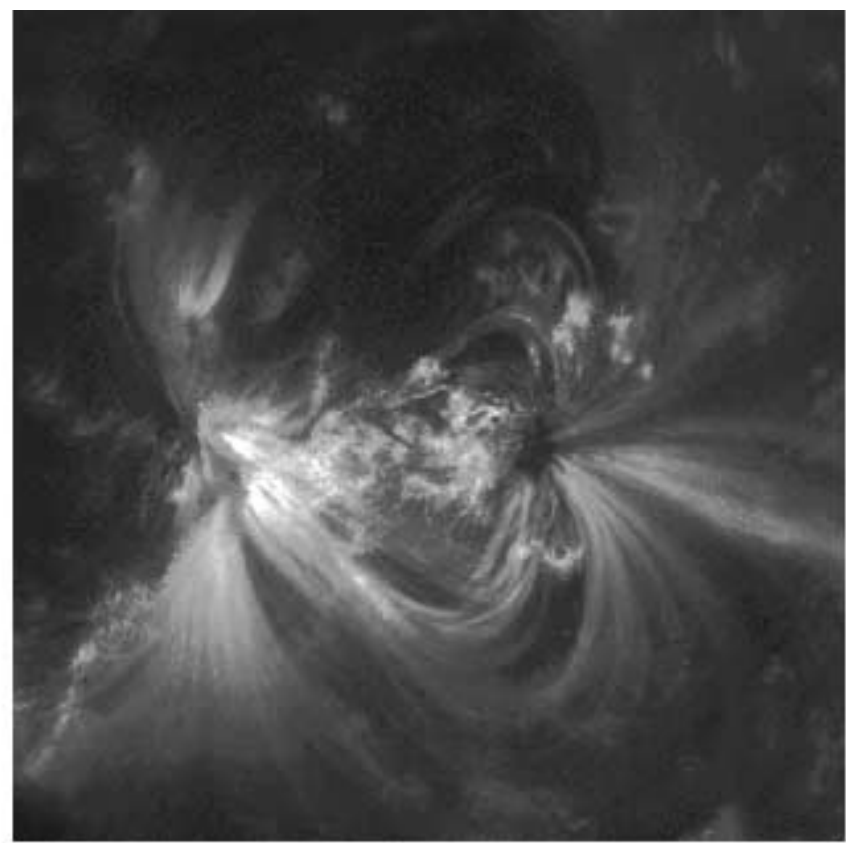

forced turbulence (Nordlund et al. 1992; Tao, Proctor, \& Weiss 1998).

In three dimensions, flux expulsion becomes more complicated (Tobias et al. 2001). If the convecting layer is stratified, up-down symmetry is broken and hexagonal cells are initially preferred. There is then a topological distinction between the isolated rising and expanding plume at the center of a hexagon and the network of sinking fluid that surrounds it. Drobyshevski \& Yuferev (1974) suggested that magnetic flux would therefore be pumped preferentially downward by the connected downflow network (see also Moffatt 1983). Actually, there is a much more powerful effect. Numerical simulations of stratified compressible convection all agree in showing a strong contrast between the gently rising plumes and the sinking fluid, which is focused into rapidly falling plumes at the corners in the network (Stein \& Nordlund 1989, 1998; Spruit, Nordlund, \& Title 1990; Cattaneo et al. 1991; Brummell, Clune, \& Toomre 2002). Now magnetic fields are governed by the induction equation and "feel" velocity rather than momentum density. Since the rising plumes expand, they transfer magnetic flux to the vigorous sinking plumes, which contract as they descend. Hence, magnetic fields are pumped preferentially downward. Such asymmetric turbulent pumping has indeed been demonstrated in various numerical experiments (Nordlund et al 1992; Brandenburg et al. 1996; Tobias et al. 1998, 2001; Dorch \& Nordlund 2001; Ossendrijver et al. 2002).

\subsection{Pumping in the Sun}

In a star, flux pumping has to compete with magnetic buoyancy. An isolated magnetic flux tube that is in pressure and thermal equilibrium with its surroundings will be less dense and therefore float upward (Parker 1979). Which process is more effective therefore depends on the vigor of convection and the strength of the magnetic field (as measured by the inverse of the plasma $\beta$ ). Previous calculations have in general been motivated by the dynamo problem and have focused on pumping at the base of the solar convection zone, with the aim of showing that poloidal magnetic flux can be pumped downward toward the tachocline where strong toroidal fields can be generated by differential rotation. This situation has been modeled by placing a strongly superadiabatic layer above a layer that is stably stratified, so that convective plumes can penetrate downward from the unstable region into the stable zone below. These numerical experiments confirm that flux pumping is extremely effective (Tobias et al. 1998, 2001; Dorch \& Nordlund 2001).

Our aim here is to show that pumping is also effective near the FIG. 3.-TRACE image of coronal loops connecting two sunspots, along with a white-light image of the sunspots (courtesy of the Lockheed Martin Solar and Astrophysics Laboratory). [See the electronic edition of the Journal for a color version of this figure.]

are purely kinematic, with no back-reaction from the distorted field on the motion. For instance, if an initially horizontal field is embedded in a layer of persistent two-dimensional eddies with up-down symmetry, then the magnetic flux will be expelled into a symmetrical pair of boundary layers at the top and bottom of the fluid layer, provided that the magnetic Reynolds number is sufficiently large (Parker 1963, 1979; Weiss 1966). This effect extends to inhomogeneous turbulent flows in two dimensions, where magnetic flux is pumped down the gradient of turbulent intensity (Rädler 1968; Moffatt 1983). This turbulent diamagnetism operates in both Cartesian and spherical geometry (Zel'dovich 1956; Spitzer 1957; Mestel 1998). It has also been demonstrated numerically for fully dynamical two-dimensional motion, driven either convectively or as photosphere, where three distinct scales of cellular convection are observed. The most vigorous motion is associated with the granules that are visible around the sunspot in Figure 1 and have a characteristic diameter of $1400 \mathrm{~km}$. There are also mesogranules with a scale of around $6000 \mathrm{~km}$ (Shine, Simon, \& Hurlburt 2000); these features appear in numerical simulations (Stein \& Nordlund 1989, 1998) and are apparently caused by collective interactions between the granules (Cattaneo, Lenz, \& Weiss 2001). Then there are the supergranules, with typical diameters of 20,000-30,000 km, which can be followed for long periods from space (Duvall \& Gizon 2000; Shine et al. 2000). Helioseismology indicates that they are at least $8000 \mathrm{~km}$ deep (Kosovichev, Duvall, \& Scherrer 2000), and they apparently correspond to a different scale of convection (Lawrence, Cadavid, \& Ruzmaikin 2001).

There is as yet no consensus on the deep structure of convection in the Sun or on the nature of the relationship 


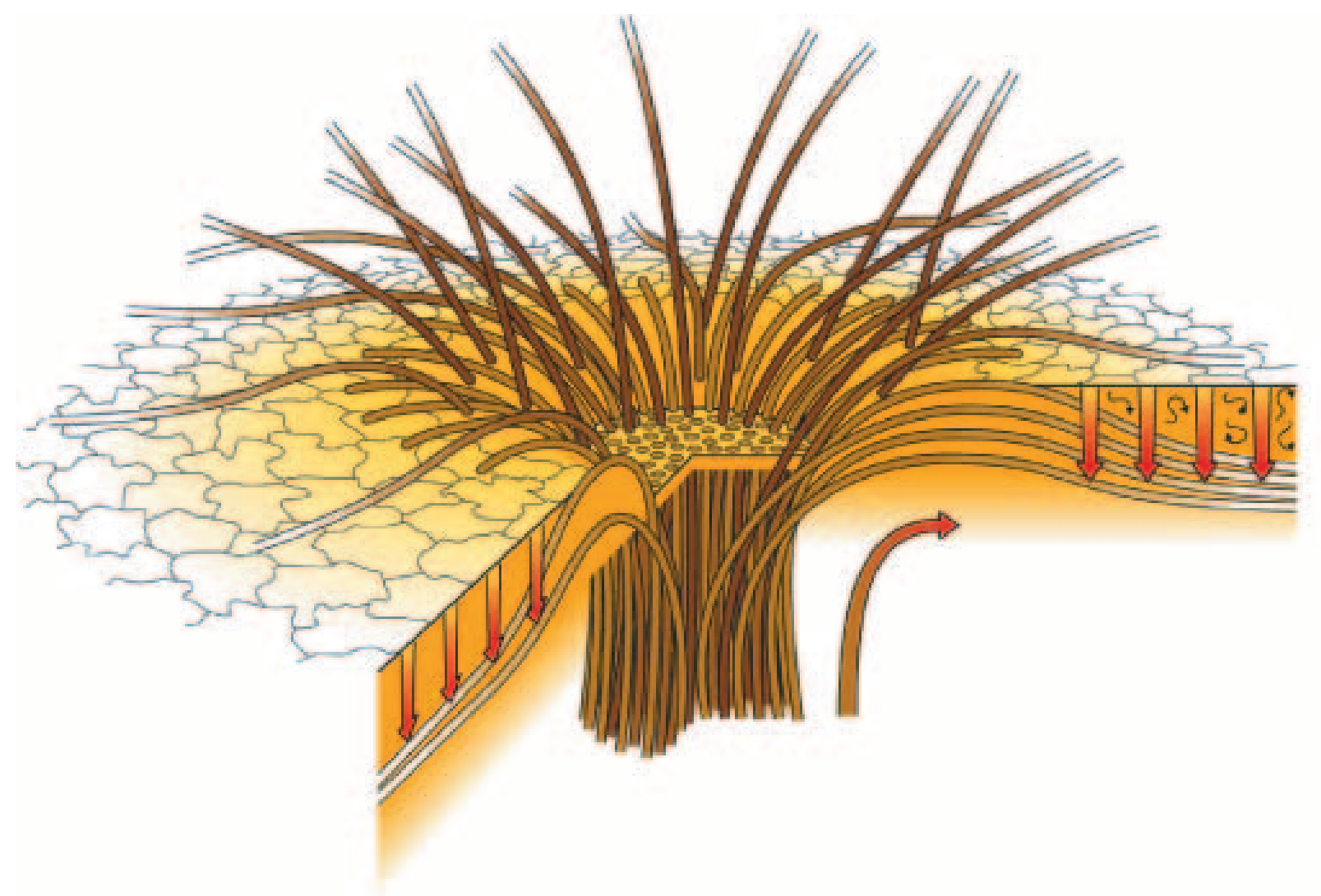

FIG. 4.-Sketch showing the interlocking-comb structure of the magnetic field in the filamentary penumbra of a sunspot (from Thomas et al. 2002b, with modifications courtesy of Eric Priest). The bright radial filaments, where the magnetic field is inclined (at about $40^{\circ}$ to the horizontal in the outer penumbra), alternate with dark filaments in which the field is nearly horizontal. Within the dark filaments, some magnetic flux tubes (i.e., bundles of magnetic field lines) extend radially outward beyond the penumbra along an elevated magnetic canopy while other, "returning" flux tubes dive back below the surface. The sunspot is surrounded by a layer of small-scale granular convection (squiggly arrows) embedded in the radial outflow associated with a long-lived annular supergranule (the moat cell; large curved arrow). The submerged parts of the returning flux tubes are held down by turbulent pumping (vertical arrows) by the granular convection in the moat.

between the small-scale granular and mesogranular patterns and the larger scale supergranules. One hypothesis is that the horizontal scale of convection increases continuously with depth as a result of self-similar merging of the sinking plumes (Stein \& Nordlund 1989). Another is that supergranules represent the preferred convective scale near the surface, with granules and mesogranules as a strongly superadiabatic turbulent boundary layer. For our purposes it suffices to adopt the latter picture. We assume therefore that there is a clear distinction between a shallow, strongly unstable layer with vigorous small-scale convection, dominated by rapidly sinking plumes, and a deeper, weakly unstable layer with more placid large-scale motion.

The moat cell that surrounds a well-developed sunspot is a large, anchored supergranule with a radial outflow that carries the smaller granules and mesogranules with it. Around the penumbra itself there is a narrow ring or "collar," with a higher outward velocity, which can be found even if the moat cell is absent (Shine et al. 1994; Shine \& Title 2001). This is associated with an upflow at the edge of the penumbra. (There may also be a slender counterflow cell that contains the sunspot flux tube below the surface, as suggested by Hurlburt \& Rucklidge 2000.) We expect that the vigorous sinking plumes in granules and mesogranules in the moat will overcome magnetic buoyancy and pump magnetic flux downward. In the lower supergranular region, magnetic buoyancy and curvature forces become relatively more important and pumping is less effective. Thus, we should expect the moat flow to be relatively neutral, allowing magnetic flux to accumulate below the upper layer. This configuration can therefore be modeled by placing a strongly unstable layer (representing the granular and mesogranular stratification) above a very weakly superadiabatic layer that represents the stratification in the moat cell. Then a horizontal field can be inserted and its evolution can be followed.

\section{MODEL CALCULATIONS}

In this section we describe numerical experiments designed to demonstrate the effectiveness of magnetic pumping. It should be stressed that the calculations included here are designed to investigate the physical processes believed to play an important role around the penumbrae of sunspots. They are not intended to represent every aspect of sunspot dynamics, including geometry and large-scale flows. For this reason the model we describe is deliberately designed to be the simplest possible, while still retaining the important physics. We begin by describing briefly the setup and geometry of our threedimensional configuration, including the equations that are to be solved and their method of solution. The model discussed here is similar to that of Tobias et al. (2001), although the latter was designed to investigate the interaction between magnetic fields and convection at the base of the solar convection zone. The reader is directed to that paper for further details. 


\subsection{Setting Up the Model}

We consider an idealized model with a cuboidal local computational domain containing a fully compressible, electrically conducting gas, confined between two horizontal, impenetrable, stress-free boundaries. This domain represents a local region of plasma located just below the solar surface. In nondimensional Cartesian coordinates, the extent of the domain is given by $0 \leq x \leq x_{m}, 0 \leq y \leq y_{m}$, and $0 \leq z \leq z_{m}$, with $x$ and $y$ being the horizontal directions and the $z$-axis pointing vertically downward. The physics is simplified by taking a perfect gas with $\gamma=5 / 3$ and assuming that the specific heats, the shear viscosity, the electrical conductivity, and the gravitational acceleration are all constant. The nondimensionalization is based on the surface values of the reference polytropic static state, together with lengths measured in terms of the depth of a fiducial upper layer, times measured in relation to an isothermal sound crossing time, and magnetic fields normalized with respect to an imposed field strength $B_{0}$. Here we consider convective motions on a scale smaller than the Rossby radius of convection, and, for this reason, rotation is not included in the model; the effect of rotation on transport of magnetic flux has been considered by Tobias et al. (2001) and Ossendrijver et al. (2002).

The static nondimensional polytropic state is given by

$$
T=(1+\theta z), \quad \rho=(1+\theta z)^{m}, \quad p_{g}=(1+\theta z)^{m+1},
$$

where $\theta$ is the nondimensional heat flux of the polytrope and $m$ is its polytropic index. For the choice of nondimensionalization described above, the equations for the temporal evolution of the velocity $\boldsymbol{u}=(u, v, w)$, the magnetic field $\boldsymbol{B}=\left(B_{x}, B_{y}, B_{z}\right)$, the density $\rho$, the temperature $T$, the gas pressure $p_{g}$, and the total pressure $p_{t}$ become

$$
\begin{gathered}
\partial_{t} \rho+\nabla \cdot(\rho \boldsymbol{u})=0, \\
\partial_{t}(\rho \boldsymbol{u})+\nabla \cdot(\rho \boldsymbol{u} \boldsymbol{u}-\alpha \boldsymbol{B B})=-\nabla\left(p_{t}\right)+\rho \theta(m+1) \hat{z} \\
+\sigma C_{k}\left[\nabla^{2} \boldsymbol{u}+\frac{1}{3} \nabla(\nabla \cdot \boldsymbol{u})\right], \\
\partial_{t} T+\nabla \cdot(\boldsymbol{u} T)+(\gamma-2) T \boldsymbol{\nabla} \cdot \boldsymbol{u}=\gamma C_{k} \rho^{-1} \nabla \cdot[\kappa(z) \nabla T] \\
+\zeta C_{k} \alpha(\gamma-1) \rho^{-1}|\nabla \times \boldsymbol{B}|^{2}+V_{\mu}, \\
\partial_{t} \boldsymbol{B}=\boldsymbol{\nabla} \times(\boldsymbol{u} \times \boldsymbol{B})+C_{k} \zeta \nabla^{2} \boldsymbol{B}, \quad \boldsymbol{\nabla} \cdot \boldsymbol{B}=0, \\
p_{g}=\rho T, \quad p_{t}=p_{g}+\frac{\alpha|\boldsymbol{B}|^{2}}{2},
\end{gathered}
$$

where $V_{\mu}$ is the rate of viscous heating, given by

$$
V_{\mu}=\frac{(\gamma-1) C_{k}}{\rho} \sigma \partial_{i} u_{j}\left(\partial_{i} u_{j}+\partial_{j} u_{i}-\frac{2}{3} \partial_{k} u_{k} \delta_{i j}\right) .
$$

Here $C_{k}$ is a dimensionless thermal diffusivity, defined at $z=0$, and $\kappa$ is the corresponding conductivity, which takes different values in the two layers; both the Prandtl number $\sigma$ (the ratio of the thermal to the viscous diffusivity) and $\zeta$ (the ratio of the magnetic to the thermal diffusivity) are also functions of $z$, and their values given here are evaluated at $z=0$. The field strength is determined by the parameter $\alpha=\sigma \zeta C_{k}^{2} Q$. Here $Q$ is the Chandrasekhar number, related to the initial field intensity $B_{0}^{2}$, the magnetic permeability $\mu_{0}$, the resistivity $\eta$, and the shear viscosity $\mu$ by $Q=B_{0}^{2} d^{2} /\left(\mu_{0} \mu \eta\right)$. The parameter $\alpha$ is related to the commonly cited plasma $\beta$ by $\beta=p_{g} /\left(0.5 \alpha B^{2}\right)$. The values of $\beta$ found in these simulations are greater than unity but are within an order of magnitude of the expected solar value.

We build a two-layer model by considering a fluid whose material properties vary rapidly with depth near an interface given by $z=1$. In particular, both the polytropic indices $\left(m_{1}\right.$ in the upper layer and $m_{2}$ in the lower layer) and thermal conductivities are allowed to differ significantly between the two layers, leading to potentially different dynamics in those layers. The stability of the polytrope in each layer to convection is determined by its polytropic index; for an adiabatically stratified layer the polytropic index is $m_{\mathrm{ad}}=3 / 2$. In our model the upper layer $(0 \leq z \leq 1)$ is chosen to be convectively unstable $\left(m_{1}=1.0\right)$ and the relative stability of the two layers is measured by the stiffness parameter, $S$, defined by

$$
S=\frac{m_{2}-m_{\mathrm{ad}}}{m_{\mathrm{ad}}-m_{1}}
$$

(Hurlburt et al. 1994). An adiabatically stratified lower layer is therefore given by $S=0$. For $S>0$ the lower layer is stable to convection, while $S<0$ signifies a lower layer that is convectively unstable. For the calculations contained in Tobias et al. (2001), designed to mimic the base of the solar convection zone, the lower layer was extremely stably stratified, with $S=$ 15 mainly. In our initial calculations designed to mimic the surface layers of the Sun (Thomas et al. 2002b), the stiffness parameter was $S=0.5$ and the lower layer was only mildly subadiabatic. Those results will be contrasted with the atmospheres chosen here, where $S=0.0$ or -0.01 , corresponding to adiabatic $\left(m_{2}=1.5\right)$ or weakly superadiabatic $\left(m_{2}=\right.$ 1.495) lower layers.

It is convenient to introduce two other nondimensional parameters in order to describe the state of the system. These are the Chandrasekhar number, $Q=\alpha / \sigma \zeta C_{k}^{2}$, which measures the strength of the imposed field $B_{0}$ relative to diffusive effects, and the Rayleigh number, Ra, which measures the supercriticality of convection in the upper layer. Since the Rayleigh number varies with depth, we evaluate $\mathrm{Ra}$ at the middle of the upper layer in the initial polytrope. Thus,

$$
\mathrm{Ra}=\frac{\left(m_{1}+1\right) \theta^{2}}{\gamma \sigma C_{k}^{2}}\left(m_{1}+1-m_{1} \gamma\right)\left(1+\frac{\theta}{2}\right)^{2 m_{1}-1} .
$$

Periodic boundary conditions are imposed on all the fields in both horizontal directions, while on the horizontal surfaces the boundary conditions are given by

$$
\begin{array}{r}
\partial_{z} u=\partial_{z} v=w=0 \quad \text { at } z=0, z_{m}, \\
T=0 \quad \text { at } z=0, \quad \partial_{z} T=\left[\frac{\kappa(0)}{\kappa\left(z_{m}\right)}\right] \theta \quad \text { at } z=z_{m} .
\end{array}
$$

These correspond to stress-free, impermeable boundary conditions for the velocity field, a constant temperature on the upper surface and a constant heat flux on the lower boundary. The boundary conditions on the magnetic field are chosen so that the horizontal components of the magnetic field vanish on the upper and lower surfaces, i.e.,

$$
B_{x}=B_{y}=0 \quad \text { at } z=0, z_{m} .
$$

The role of magnetic boundary conditions in pumping calculations was discussed extensively by Tobias et al. (2001); 
these boundary conditions allow magnetic flux to escape either upward or downward from the domain, and the calculation therefore takes the form of a run-down experiment for the magnetic flux.

The equations together with the boundary conditions described above are integrated numerically as an initial value problem using a mixed finite-difference pseudospectral scheme. The equations are discretized and then advanced in time using an explicit three-level Adams-Bashforth scheme for the nonlinear terms and an implicit Crank-Nicolson scheme for the linear terms. Details of the numerical scheme can be found in Tobias et al. (2001). It should be noted that these are far from trivial computations. The runs described below used a mesh of $256 \times 256 \times 350$ grid points, and each one consumed about 30,000 processor hours on a massively parallel Silicon Graphics Origin 2000 supercomputer (at the National Center for Supercomputing Applications, University of Illinois, Champaign-Urbana, Illinois).

\subsection{The Pattern of Convection}

We now describe the results of two numerical experiments designed to explore the role of pumping in, transporting, and submerging magnetic flux at the edge of a sunspot penumbra. In previous calculations that modeled the base of the solar convection zone, the highly superadiabatic convection layer lies above a lower layer that is very stably stratified. In contrast, as discussed earlier, the highly turbulent surface layers of the Sun lie above a region of both more laminar and larger scale convection. The latter convection, with a larger horizontal scale and a much longer turnover time, acts so as to produce an almost adiabatic stratification in the deeper layer below. Thomas et al. (2002b) introduced a model where the lower layer was chosen to have a mildly subadiabatic stratification. However, on the timescales of turbulent granular and mesogranular motion this deeper layer will appear adiabatic or only very weakly superadiabatic. In this subsection we investigate the role of such a lower layer in modifying the form of the convection in the upper layer. After that, we wish to ascertain whether the modified convection successfully transports magnetic flux out of the upper layer into the layer below.

We begin by investigating the properties of a purely hydrodynamic model (with no magnetic field), for two different values of the stability parameter $S$ but with all other parameters held fixed. We set $\sigma=\zeta=0.1, \theta=10, x_{m}=y_{m}=6$, and $z_{m}=2$. Thus, the upper unstable layer has an initial temperature and density contrast of 11 and the effects of compressibility are substantial. With these parameter values, the Rayleigh number is $\mathrm{Ra}=2400 / C_{k}^{2}$ and we choose $C_{k}=0.07$ so that this layer is very strongly unstable to convection, giving a Rayleigh number, $\mathrm{Ra}=4.9 \times 10^{5}$, that is many times larger than the critical Rayleigh number for the onset of convection. (These parameters in the upper layer correspond to the "turbulent" pumping case of Tobias et al. 2001.) The properties of the lower layer are controlled by the stability parameter. Here we consider two cases: $S=0.0$, corresponding to an adiabatically stratified lower layer, and $S=-0.01$, corresponding to a very weakly superadiabatic lower layer.

For these two values of the stability parameter $S$, the purely hydrodynamic equations are integrated until the solution settles down to a vigorous time-dependent flow with statistically steady properties. Volume renderings of the solutions for both $S=0.0$ and -0.01 are shown in Figure 5. This figure shows a color-coded three-dimensional image of the vertical velocity $w$ in the purely hydrodynamic solution, with cool downflows shown in blue and warm upflows in red. The volume renderings demonstrate that the solution for both parameter values takes a similar form. The general properties of the convection for both parameter values can be seen clearly from this figure. The turbulent convection in the upper layer takes the form of strong, downward-sinking plumes in a dynamic network surrounding broad, weaker upflows. It is well known (see, e.g., Hurlburt et al. 1994) that this asymmetry between the upflows and downflows is due to the stratification and compressibility of the flow and is enhanced by the presence of a lower layer of fluid (Brummell et al. 2002). The lower panels in Figure 5 show color-coded volume renderings of the enstrophy density (the square of the vorticity) on a logarithmic scale for clarity. Strong enstrophy is contained in downward-sinking plumes (shown in red and orange), which are visible in both the upper and lower layers.

The boundary between the upper and lower layer (delineated by dashed white lines in the volume renderings) can also be seen in the nature of the convection. The flows in the upper layers are very similar to those found in the upper layer of previous calculations (e.g., Brummell et al. 2002). It is in the lower layer that the presence of a weakly unstable or neutrally stable stratification has a large effect. Figure 6 compares the properties of the vertical velocity as a function of depth with those for a representative calculation with a very stable lower layer $(S=30)$ described by Brummell et al. (2002). For the stably stratified lower layers of Tobias et al. (2001) and Thomas et al. (2002b), the downward-sinking plumes were decelerated as they entered the lower layer as a result of the action of buoyancy braking, with the deceleration being more rapid in a more stable lower layer. Sufficiently far below the interface between the unstable and stable layers, the plumes came to rest and the vertical flux of kinetic energy, $\left\langle\frac{1}{2} \rho|\boldsymbol{u}|^{2} w\right\rangle$, dropped to zero as shown in the top panel of Figure 6 . (Here the angle brackets denote a horizontal average and the plots are further averaged over a significant portion of time during the simulation.) Below this penetration depth, the fluid was essentially static with no convection being driven. Here, with the lower layer not convectively stable, the situation is different. For both nonpositive values of the stability parameter, the downward plumes again overshoot the base of the strongly unstable region. However, since the layer below is not stable, a plume is free to continue without being braked, and thus does so, triggering further flows as it moves toward the bottom of the lower layer. The action of stratification and changes in the thermal conductivity $d o$ act to slow the progress of the plume, but substantial vertical velocities are possible throughout the lower layer until the plume is stopped by the impenetrable bottom boundary. The second panel of Figure 6 shows $\left\langle w^{2}\right\rangle$ as a function of $z$. This plot clearly demonstrates that the strongest vertical flows arise in the middle of the upper layer, but there is also a significant amount of energy in the vertical motion in the lower layer $(z>1)$, and this (like the kinetic energy flux for these cases in the top panel of Fig. 6) falls to zero only very close to the base of the domain. Clearly, we cannot meaningfully define a penetration depth for convection (Brummell et al. 2002) unless the lower layer is stably stratified.

The third panel of Figure 6, which shows $\langle w\rangle$ as a function of $z$, confirms, however, that the nature of these vertical flows is different in the upper and lower layers. In the upper layer, there is a strong asymmetry between the upflows and downflows, with the downflows being stronger, more dense, and narrow while the upflows are broad, less dense, and slow. Since 


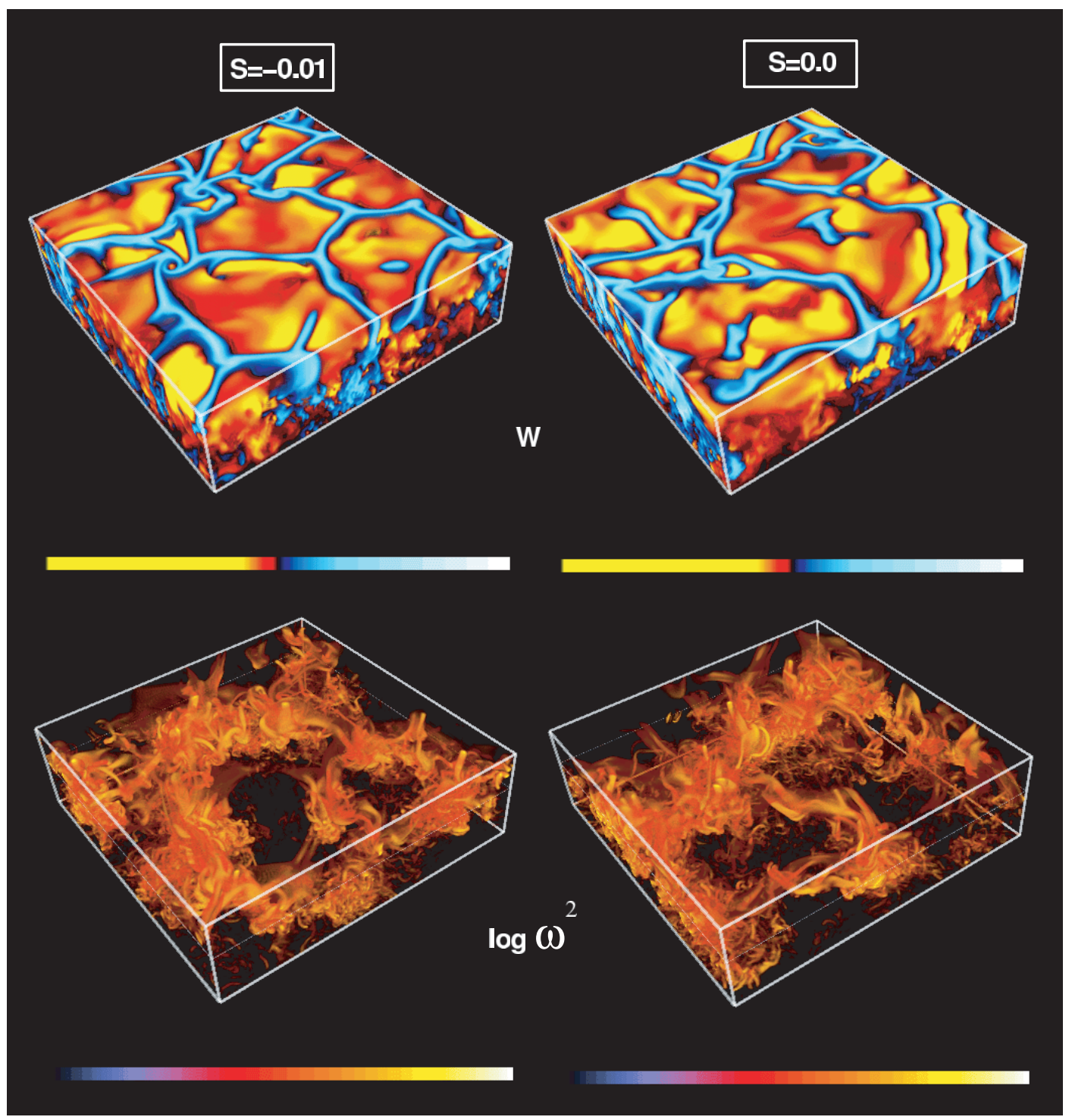

FIG. 5.-Volume renderings of the instantaneous vertical velocity $w$ and the logarithm of the enstrophy density $\omega^{2}$ for fully developed, purely hydrodynamical $(\boldsymbol{B}=0)$ penetrative convection, for two values of the stability parameter, $S=0.0$ and -0.01 . In the volume renderings, each value is assigned a color and an opacity. Color tables are as shown (warm red-orange colors depict upflows, and cool blue-white colors represent downflows; bright red-yellow-white colors exhibit strong enstrophy density). Strong values are opaque, while weak values are translucent.

$\langle\rho w\rangle=0$ in a steady state, this leads to a clear net vertical upward velocity in the upper layer. The asymmetry between upflows and downflows is further demonstrated by the plots of $\left\langle w^{3}\right\rangle$ as a function of $z$ in the bottom panel of Figure 6: because of the dominance of vigorous sinking plumes, $\left\langle w^{3}\right\rangle$, like the kinetic energy flux, is positive throughout the domain. In the lower layer, however, $\langle w\rangle$ decreases rapidly toward zero as $z$ increases past unity. This is because, although there are substantial vertical flows in this region, the density varies much less than in the upper layer. Because the convection is locally only weakly supercritical, the flows are much more symmetric and cellular in this lower layer. Figure 7 shows $w(x, y)$ averaged vertically over first the upper and then the lower layer. In the upper layer it is clear that there is a network of downflows defining the boundaries of the upflows, with about six or seven convection cells in the computational domain. The pattern of convection in the lower layer apparently corresponds to the additional presence of a single mesocell in the upper layer (Stein \& Nordlund 1998; Cattaneo et al. 2001). The presence of this change of scales is not entirely obvious from a power spectrum of the flows at different depths (Fig. 7b). No new peak is apparent in the power spectrum at depths in the lower layer, although in general the power has increased at lower wavenumbers.

We shall see that the change in flow pattern of the lower layer compared to the upper has important consequences for the transport of magnetic flux in this region. It should be stressed again that there is very little difference in the hydrodynamic properties of the flows between the adiabatic and weakly superadiabatically stratified lower layers. In both cases the flows are apparently driven by the dynamics in the strongly unstable upper layer. We note also another property of these solutions, although one that does not have an immediate effect on the transport of magnetic flux, namely, the presence of significant horizontal flows near both the top and bottom boundaries of the domain. Further investigation of the nature of these flows reveals strong mean flows along the stress-free bottom boundary, driven by the variable temperature that is 

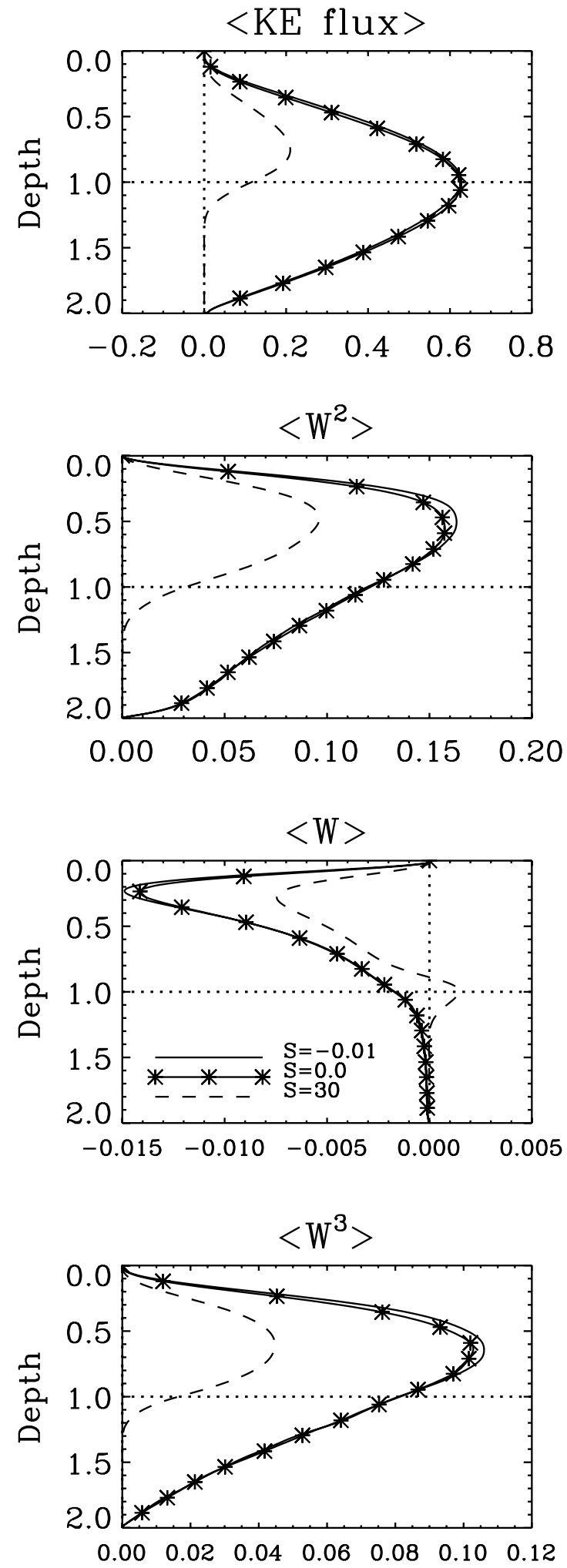

Fig. 6.-Horizontally averaged values of the kinetic energy flux, the vertical velocity $w$, the square of the vertical velocity, and the cube of the vertical velocity for the two hydrodynamic calculations shown in Fig. 5 plus a simulation with a very stable lower layer $(S=30$ from Brummell et al. 2002) for comparison. allowed by the fixed-flux boundary condition. Similar behavior has been found by Robinson et al. (2003).

\subsection{Magnetic Pumping}

Having established a statistically steady convecting state, we wish to identify its transport properties when magnetic flux is added. To achieve this, we insert a thin sheet of horizontal magnetic field $\left(B_{x}=0, B_{y}=B_{0}\right)$ between $z=0.55$ and 0.60 in the middle of the upper layer (as in Tobias et al. 1998, 2001). In order to keep the total pressure, $p_{t}$, continuous between the layer of magnetic field and the surrounding field-free fluid, the density is decreased within the magnetized layer, while the temperature remains continuous. The local decrease in density leads to the development of instabilities driven by magnetic buoyancy. These instabilities combine with convectively driven motion to produce strands of locally strong magnetic fields. Magnetic buoyancy then competes with advection of magnetic flux by convective flows in the subsequent evolution of the magnetic and velocity fields.

For the calculations described below, $B_{0}$ is chosen so that the Chandrasekhar number is initially fixed at $Q=10^{5}$ within the thin magnetic layer. (This is the strongest initial field that can be accommodated in the model setup without requiring the density in the layer to drop unphysically below zero, and this large value is chosen since pumping would be most difficult in this case.) Figure 8 gives volume renderings of the magnetic energy density and vertical velocity near the beginning and end of the calculation for $S=-0.01$ (the figures for $S=0.00$ have a very similar form). It is clear that a significant redistribution of the magnetic energy has occurred. At early times, the horizontal layer of magnetic field has merely begun to rise by the action of magnetic buoyancy, although some crinkling due to advective effects can already be seen. At later times, a statistically steady state exists where the majority of the magnetic field is confined to the lower layer. The advective action of the strong downflowing plumes has dominated and dragged the magnetic field down. In the process, the fields have been stretched and amplified by the advection and by the strong vortical nature of the plumes, especially where they are decelerated. The result is that the strongest magnetic field is concentrated around the plumes in the lower layer. Notice that this is not a static situation: strong elements of field are rising through magnetic buoyancy all the time, but that field is eventually caught in the downflows and recirculated back to the lower layer. This behavior is similar to that observed in Tobias et al. (2001), and thus downward magnetic pumping appears to remain a robust feature of the upper convective layer even when the lower layer also is unstable (or neutrally stable) to convection.

This process can be analyzed in detail using various measures described by Tobias et al. (2001). The initial transport of magnetic flux is clearly visible in Figure 9, which shows vertical profiles of horizontal averages of the $y$-directed magnetic field $\left\langle B_{y}\right\rangle$ at successive times as the calculations progress. For both cases, the maximum of $\left\langle B_{y}\right\rangle$, initially located at around $z=0.575$, at first rises and then migrates toward a more steady position below $z=1$. The initial rise and subsequent migration downward of magnetic flux is perhaps more clearly demonstrated by the color-coded spacetime diagram for the transport of magnetic flux in Figure 10, which charts the evolution of $\left\langle B_{y}\right\rangle$ as a function of time and depth. For both cases, the evolution is similar: an initial rise of the flux due to the combination of magnetic buoyancy and advection by 

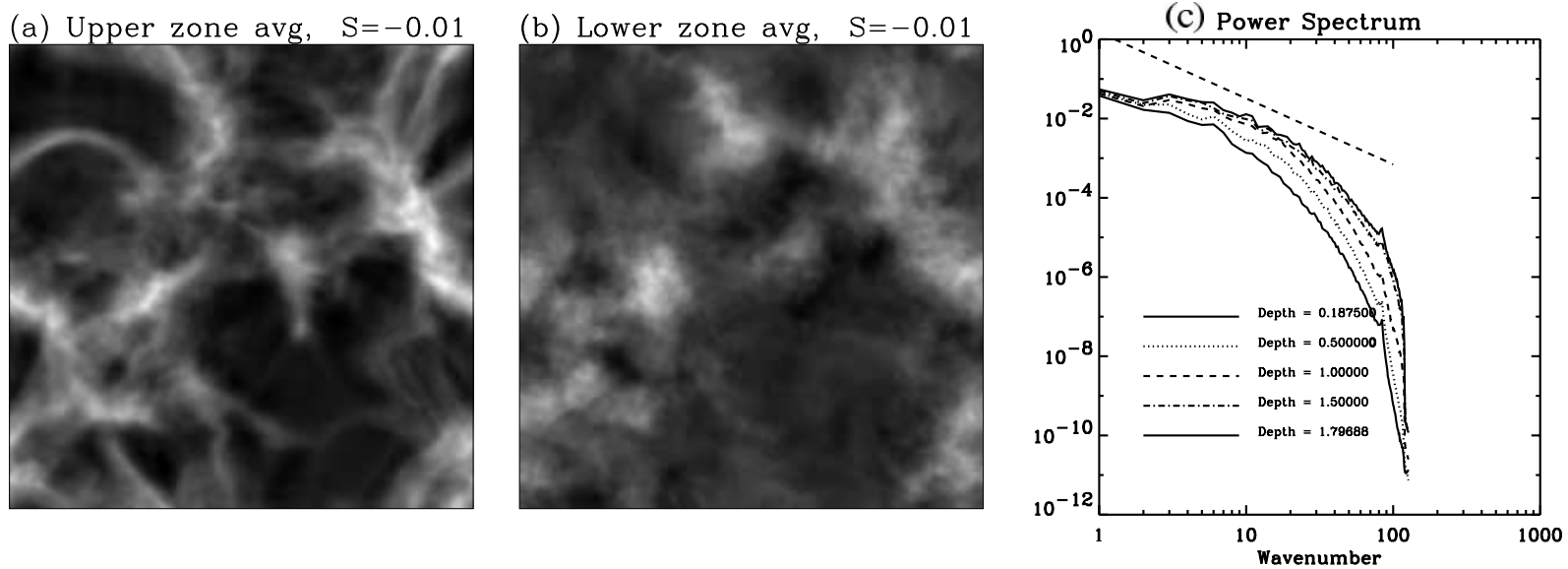

FIG. 7. - Gray-scale plots of the horizontal distribution of the vertically averaged values of the instantaneous vertical velocity $w(x, y)$ in the $(a)$ upper $(0 \leq z \leq 1)$ and $(b)$ lower $(1 \leq z \leq 2)$ layers for the purely hydrodynamical calculation with $S=-0.01$. Light tones indicate downflow; dark tones, upflow. (c) Power spectra of the flows at various depths.

the upflows (shown by the white and red ridge that runs diagonally from the imposed position of the initial flux to the top of the box) is followed by a redistribution of the flux, largely due to advection by the downflows.

Figure 11 shows the distribution of flux for the $S=0.0$ and -0.01 simulations performed in this study, together with that for the calculation with a mildly stable lower layer $(S=0.5)$, described by Thomas et al. (2002b), all at later stages during their evolution, well after the initial buoyant rise and subsequent dominant pumping phase. By this stage, all the calculations have flux distributions that peak in the lower layer below the interface and that have a significant proportion of the remaining flux residing in the lower layer, as did the earlier models of Tobias et al. (2001). In each case, the strong

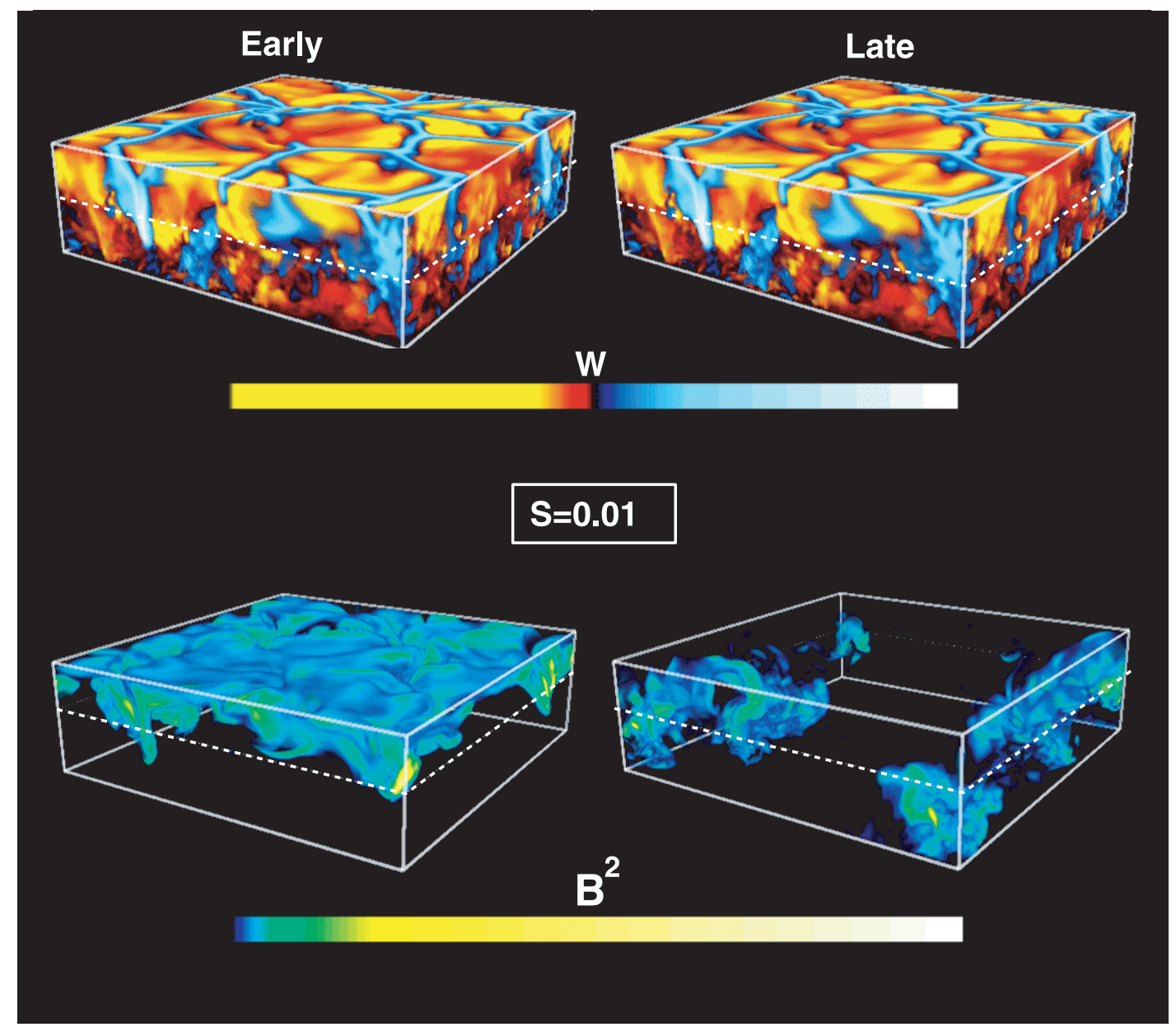

FIG. 8.-Volume renderings of the instantaneous vertical velocity $w$ and the magnetic energy density $B^{2}$ at stages near the beginning and near the end of the

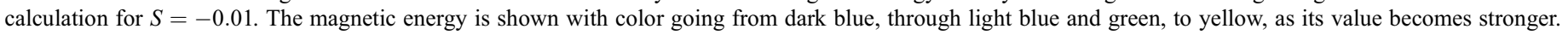



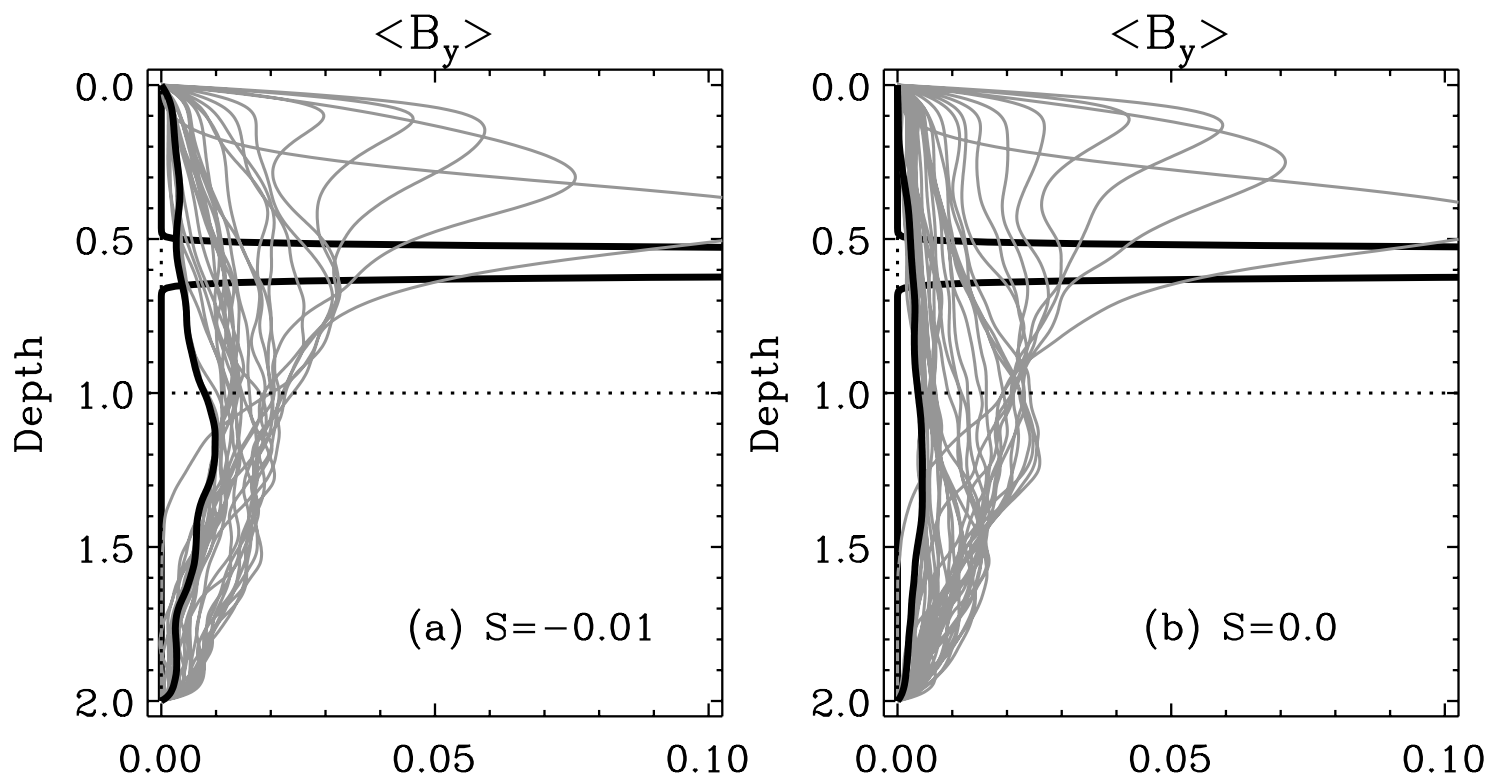

FIG. 9.- Horizontally averaged values of the magnetic field component $B_{y}$ as functions of depth at uniformly spaced times over the course of the flux-pumping simulations with $S=-0.01$ and 0.0 . The initial and final states are shown as thick lines.

downward-sinking plumes have advected magnetic flux into the lower layer. However, the nature of the lower layer significantly affects the next phase of the evolution and therefore the ultimate flux distribution. For the case $(S=0.5)$ in which the lower layer is stable, the strong motions are limited by the penetration depth (around $z=2.5$ in this case), and therefore magnetic flux does not reach the lower boundary (at $z=3.5$ ) at this stage. For the cases $(S=0.0$ and -0.01$)$ in which the lower layer is not stable and therefore more active, the continued (but different) action of the convective motion in the lower layer continues to pump and redistribute the magnetic flux. This can be seen easily in Figure 11 since the flux distributions for these cases are significant near the lower boundary of these simulations (at $z=2$ ). When $S \leq 0$, the vertical motion in the lower layer is still reasonably strong $\left(\left\langle w^{2}\right\rangle\right.$ is still large; Fig. 6) with the downflows dominating $\left(\left\langle w^{3}\right\rangle\right.$ positive), although there is little significant asymmetry between the strengths of the upflows and downflows $(\langle w\rangle \sim 0)$. Once magnetic flux has reached the lower layer, it is transported by the generally more symmetric, slower convection (after allowing for the structure produced by advection), while more flux is continually added from the upper layer. However, regardless of the details, a significant redistribution of the magnetic flux has occurred in all cases, removing much of the magnetic field from the upper layer.

It should be noted that our choice of magnetic boundary conditions allows flux to leave the computational domain. Thus, a significant amount of the horizontal flux is lost through the top of the box. By the end of the calculation, if the flux has also been carried as far as the lower boundary, some of it has also been lost through the bottom of the box. In other words, the simulation is of the "run-down" variety. The flux distribution that develops therefore eventually decays in a self-similar manner, but generally with more flux in the lower (a) $\mathrm{S}=-0.01$

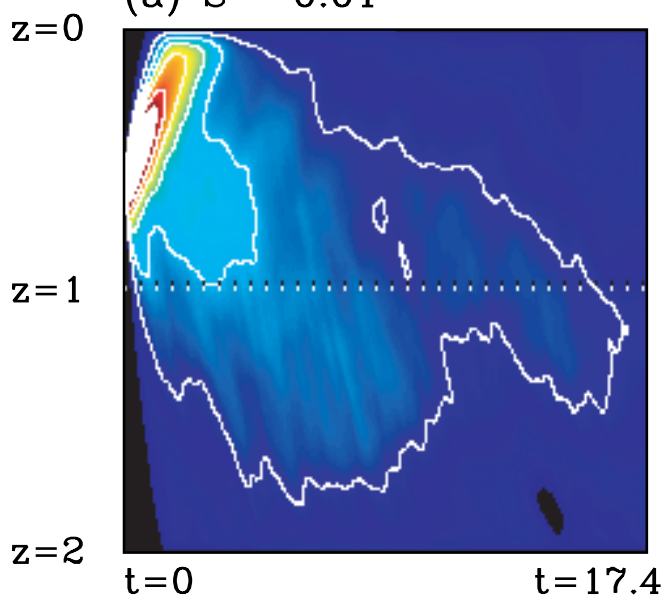

(b) $S=0.0$

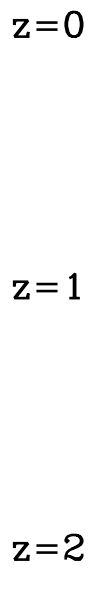

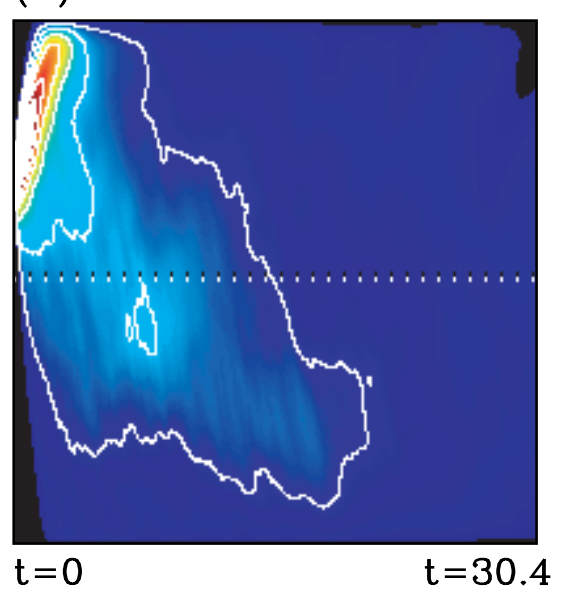

FIG. 10.-Color-coded spacetime diagrams showing the redistribution of magnetic flux for the two calculations $(S=-0.01$ and 0.0$)$. The horizontally averaged value of $B_{y}$ is shown as a function of depth $z$ and time $t$, with increasing magnetic field strength indicated by color in the order black-blue-green-yellow-red-white. The horizontal dotted line indicates the boundary between the upper and lower layers of the model. 


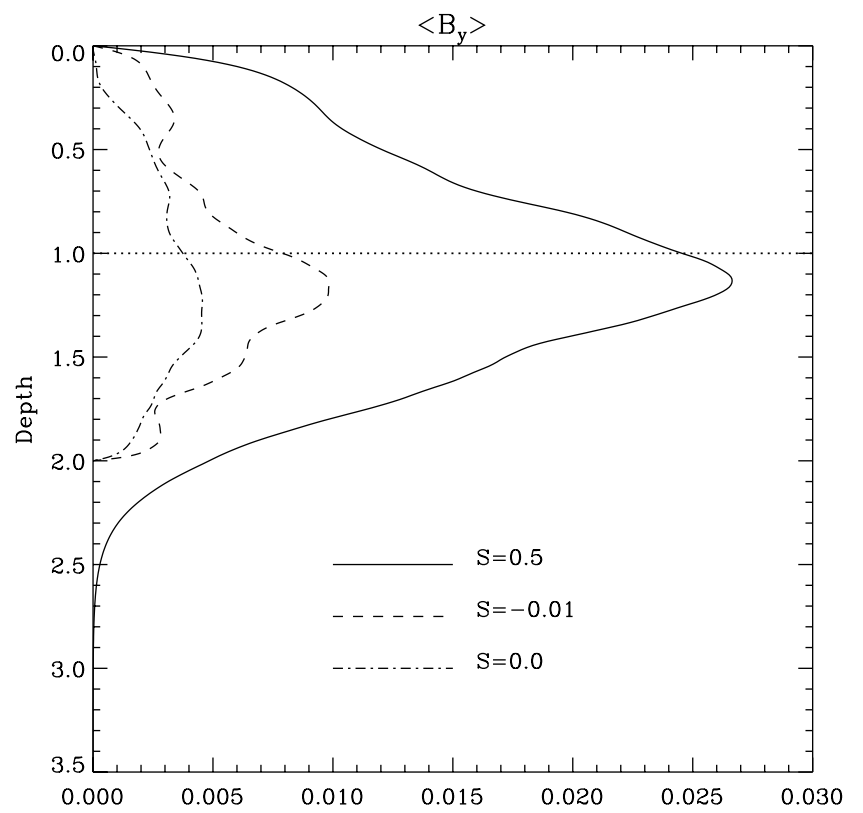

FIG. 11.-Horizontally averaged values of the magnetic field component $B_{y}$ as functions of depth at the final state of each of three calculations: a mildly stable lower layer $(S=0.5$, from Thomas et al. 2002b), a neutrally stable lower layer $(S=0.0)$, and a weakly unstable lower layer $(S=-0.01)$.

than in the upper layer. In cases in which the lower layer is convectively stable (e.g., the $S=0.5$ case shown), magnetic flux can only leak down below the penetration depth through the action of diffusion. Here (cases $S=0.0$ and -0.01 ) the magnetic field in the lower layer is further transported by convection by pumping processes on an advective timescale for the slower motion in the lower layer. The peak in the distribution does, however, appear to remain just below the interface in all cases.

The run-down nature of these simulations means that it would be somewhat confusing to add absolute values, for instance, of the peak magnetic energy in Figure 8, since the later values will be significantly smaller as a result of the flux losses. A more meaningful measure is the proportion of remaining magnetic flux (i.e., the magnetic flux that has not been lost from the computational domain) in the upper and lower layers, and this is shown in Figure 12. This figure shows that the evolution of the flux distribution is very similar for the two cases considered here. In both cases, flux is rapidly transported, so that by about 6 sound crossing times (corresponding to approximately one turnover time of the vigorous convection) more than $50 \%$ of the remaining flux resides in the lower layer. This timescale is similar to that for calculations with a stable lower layer; up to this stage, the presence of a convecting lower layer seems to make little difference to the dynamics of the magnetic flux. Later, however, magnetic flux continues to be redistributed by convection in the lower layer as it is expelled from the upper layer by the downward turbulent plumes. This process is complicated by events such as the one visible in the $S=-0.01$ case between $t=10$ and 15 . The redistribution of magnetic flux by advection and stretching acts in competition with magnetic buoyancy, but by this stage the magnetic field there is relatively weak and so the effects of magnetic buoyancy are presumably small. Hence, even the weak convection in the lower layer is able to transport this flux efficiently.
To sum up, what we have demonstrated here and in our earlier calculations is that magnetic pumping is a robust phenomenon. After an initial phase, in which magnetic flux escapes through the upper boundary, the vigorous downwardsinking plumes in the strongly superadiabatic layer redistribute magnetic flux and expel it into the layer below. This occurs whether the layer below is mildly subadiabatic $(S=0.5)$, as in the model of Thomas et al. $(2002 \mathrm{~b})$, or adiabatic $(S=0.0)$ or weakly superadiabatic $(S=-0.01)$, as in the runs presented here. In each case the details of the dynamics are different, but the mechanism for flux transport remains essentially the same.

This mechanism relies on turbulent transport of mean magnetic fields by convective motions, as a result of correlations between small-scale magnetic fields and small-scale flows. Physically these correlations occur as a result of asymmetry in the convection, where broad expanding upflows are surrounded by a dynamic network of contracting sinking plumes. Compressibility and stratification lead not only to the peak velocities in the downflows being larger than those in the upflows but also to preferential advection of the small-scale fields into the downflows and hence a significant correlation. Mathematically, this effect can be seen on examining the induction equation (5) in a compressible flow, which can be rewritten as

$$
\partial_{t} \boldsymbol{B}+(\boldsymbol{u} \cdot \nabla) \boldsymbol{B}=(\boldsymbol{B} \cdot \nabla) \boldsymbol{u}-\boldsymbol{B}(\nabla \cdot \boldsymbol{u})+C_{k} \zeta \nabla^{2} \boldsymbol{B} .
$$

For turbulent flows, the most significant term governing the evolution of the magnetic field is the advective term $\boldsymbol{u} \cdot \nabla \boldsymbol{B}$. To determine the evolution of the mean (i.e., horizontally averaged) magnetic field, it is instructive to decompose the magnetic and velocity fields into their mean and fluctuating parts (in the same spirit as in mean field dynamo theory). If we set $\boldsymbol{u}=\langle\boldsymbol{u}\rangle+\boldsymbol{u}^{\prime}$ and $\boldsymbol{B}=\langle\boldsymbol{B}\rangle+\boldsymbol{B}^{\prime}$, where again the average is taken horizontally, then the advective term has a large-scale (laminar) contribution given by $(\langle\boldsymbol{u}\rangle \cdot \nabla)\langle\boldsymbol{B}\rangle$ and a contribution from the mean interaction of small-scale field and smallscale flow given by $\left\langle\left(\boldsymbol{u}^{\prime} \cdot \nabla\right) \boldsymbol{B}^{\prime}\right\rangle$. For turbulent flows (at high

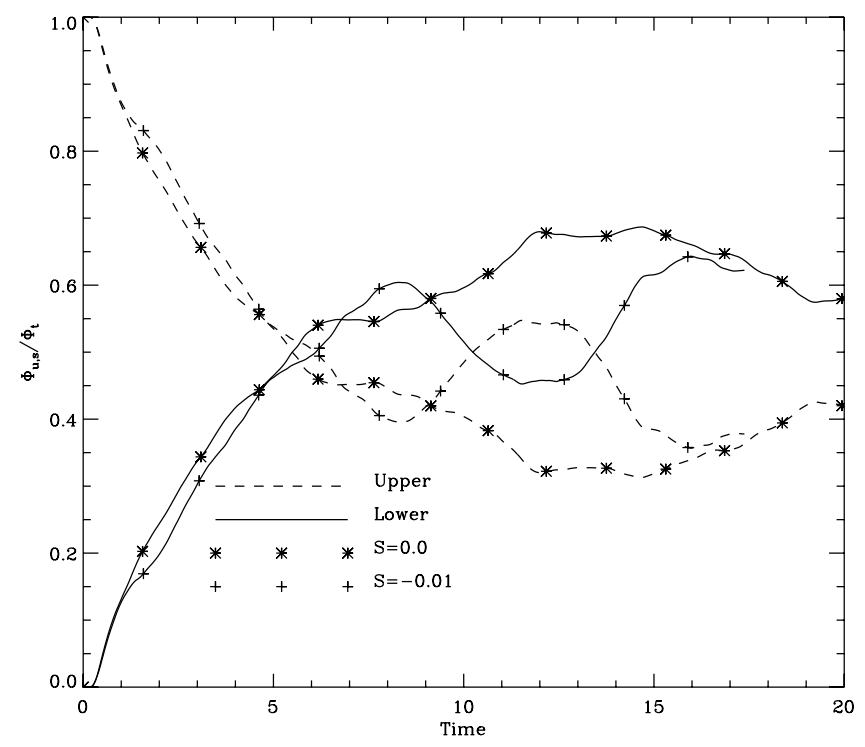

FIG. 12.-Proportions of the remaining magnetic flux residing in the upper and lower layers as functions of time for the calculations with $S=-0.01$ and 0.0 . 
Reynolds and magnetic Reynolds numbers) this second contribution dominates. Transport therefore depends on the correlations between small-scale fields and flows. The physical argument outlined above provides a mechanism for correlating the small-scale fields with the downflows, and so the turbulent transport will be dominated by these flows, hence the net downward transport of magnetic fields.

We have found through this and the preceding sets of simulations that turbulent convection can hold down a remarkably strong field, with intensity up to the order of equipartition with the peak kinetic energy of the flow. For solar surface granulation then, with a maximum convective velocity of at least $2-3 \mathrm{~km} \mathrm{~s}^{-1}$ and a density (at $1000 \mathrm{~km}$ depth) of $3 \times 10^{-6} \mathrm{~g} \mathrm{~cm}^{-3}$, we would expect pumping to be able to hold down a magnetic field of at least $1500 \mathrm{G}$. It should be remarked again, however, that this is an average effect. The distribution of flux that is maintained, with a peak just below the interface, is not a static equilibrium. Rather, magnetic field is coming and going at all times to maintain this average. Pumping is continually operating and elements of the magnetic field are continually escaping the lower layer by magnetic buoyancy. These escaping elements tend to be the strongest, most concentrated fluctuations in the field and may therefore often be identified as rising magnetic flux tubes or moving magnetic features (see $\S 7$ ).

We have perforce adopted a highly idealized configuration for these numerical experiments. The structure of the field around a sunspot penumbra, as sketched in Figure 3, is very different, since the field lines are anchored at one end to the flux tube that lies below the spot. As a result, flux pumping has to compete not only with magnetic buoyancy but also with magnetic curvature forces (caused by tension along the field lines). Although we have not attempted to model this more complex field geometry, our models support the idea that turbulent granules and mesogranules are able to drag magnetic flux down below the solar photosphere. Since the speed of convection decreases with depth beneath the surface, the sinking plumes would become too weak to transport the magnetic flux eventually and a balance between advection and buoyancy might be reached. Moreover, the outward flow in the moat cell requires a large-scale upflow around the spot, which must limit the extent to which the flux in dark penumbral filaments can be pumped downward. We expect therefore that flux pumping should lead to submerged fields with the form suggested in Figure 4. Moreover, this physical process plays an important role in determining the global structure of a sunspot.

\section{CONVECTION IN THE PENUMBRA}

In this section we attempt to interpret features observed in the penumbra in the light of our theoretical understanding of magnetoconvection (Hurlburt, Matthews, \& Rucklidge 2000; Weiss 2002). Most nonlinear studies have been concerned with the effect of an imposed vertical magnetic field on convection (e.g., Weiss, Proctor, \& Brownjohn 2002), which is appropriate for modeling umbral convection but not for the penumbra. With a vertical field there is a magnetically dominated regime in which the cells are very slender and the plumes are closely spaced. In the parameter range relevant to a sunspot umbra, there will only be small-scale convection; we expect to find a pattern of aperiodic spatially modulated oscillations, which can explain the existence of umbral dots. So long as the imposed field $\boldsymbol{B}_{0}$ is vertical there can be no preferred horizontal direction and the convection pattern can remain stationary in space. Once $\boldsymbol{B}_{0}$ is inclined, this degeneracy is broken. The tilt then defines a horizontal direction in the vertical plane that contains $\boldsymbol{B}_{0}$. This loss of symmetry implies that convection in a stratified layer has to set in as a pattern of traveling waves (Matthews et al. 1992). In two dimensions, the direction in which the waves travel is found to depend sensitively on the inclination and strength of the imposed field and also on the vigor of convection (Hurlburt, Matthews, \& Proctor 1996). Hurlburt et al. (2000) obtained three-dimensional numerical solutions and found that the pattern changed as the inclination was increased. With a modest inclination $\left(22^{\circ}\right)$ there was a modulated traveling wave: the pattern of spatially modulated oscillations drifted away from the direction of tilt. As the inclination was increased, the cells developed into distorted rolls, still drifting in the same direction. In a strong horizontal field, rolls with their axes along the field are naturally preferred (Danielson 1961).

Within a sunspot, it is possible that there may be some interchanges between fields with different inclinations at the umbral-penumbral boundary, or even sporadic episodes of reconnection within the penumbra itself. Nevertheless, as we saw in $\S 1$, the overall geometry of the magnetic field precludes significant interchanges between bright and dark penumbral filaments. Hence, each family must be considered separately. The bright filaments have typical widths of around $300 \mathrm{~km}$ and lifetimes of order an hour. Detailed observations show that they are made up of separate, elongated, bright grains (Muller 1992). These grains travel inward in the inner penumbra, penetrating into the umbra itself, but travel outward in the outer penumbra (Lites et al. 1998; Sobotka, Brandt, \& Simon 1999; Sobotka \& Sütterlin 2001; Scharmer et al. 2002). We interpret the bright grains as hot rising plumes but regard their apparent motion as a traveling wave phenomenon, rather than as due to actual radial inflows or outflows of the plasma. It follows that the penetration of bright filaments into the umbra is associated with a migrating pattern of convection and not with material motion. The reversal of the direction in which the bright grains move can be ascribed to the increasing tilt of the magnetic field with increasing radius.

In the dark filaments, where the field is strongly inclined (to the vertical) and almost horizontal at the outer edge of the spot, we expect to find radially oriented convection rolls. Here there is more likely to be overturning motion, which may be either quasi-steady or oscillatory. The observations show that this form of convection is relatively inefficient. Model calculations, involving an asymptotic treatment of magnetoconvection in very narrow cells (Julien, Knobloch, \& Tobias 2000, 2003), indicate that a number of transitions may occur as the inclination angle of the magnetic field is increased. Predominant among these is a sharp transition to a qualitatively different, and much less efficient, mode of convection as the field becomes more inclined. This transition occurs both for convection rolls in the plane of the inclined magnetic field and for those whose axis is perpendicular to this plane. This transition to a "horizontal field" branch is suggestive but has yet to be demonstrated in a fully consistent model where the horizontal scale of convection arises naturally.

It is instructive to compare our description of penumbral convection with one in which an isolated flux tube is allowed to move in an axisymmetric background field (Schlichenmaier, Jahn, \& Schmidt 1998a, 1998b; Schlichenmaier 2002). This approach is based on a picture of interchanges between the bright and dark filaments and cannot therefore represent them as 
two families of field lines that are permanently distinct. In these calculations, a thin flux tube is embedded in a magnetohydrostatic sunspot model, starting in contact with the outer boundary of the spot. As the tube heats up, it moves inward and plasma flows upward and outward along it. The flux tube maintains its identity throughout the calculation, whereas convection is a spatially continuous process. The results can, however, be related to the upward phase of a spatially modulated oscillation and it would be possible to represent the complementary downward motion as well.

\section{THE EVERSHED FLOW}

As discussed in $\S 2$, most of the photospheric Evershed flow runs along the returning penumbral flux tubes and hence may be intimately connected with the flux-pumping mechanism that submerges these flux tubes. The flux-pumping scenario is quite consistent with the siphon flow theory of the Evershed flow, in which a flow is driven along an arched flux tube by a pressure drop between the two footpoints of the arch (Meyer \& Schmidt 1968; Thomas 1988; Montesinos \& Thomas 1997; Schlichenmaier et al. 1998a, 1998b). Indeed, siphon flow models for the photospheric Evershed flow have generally assumed that some unspecified force holds down the outer, submerged part of the arched flux tube against magnetic buoyancy (e.g., Thomas \& Montesinos 1990), and we now suggest that magnetic flux pumping provides this force.

A typical returning flux tube emerges in the inner or middle penumbra and returns below the surface in the outer penumbra or just beyond the outer penumbral boundary. For a thin flux tube in equilibrium, the total pressure (gas plus magnetic) inside the flux tube must balance the total pressure just outside the flux tube. If we consider the two footpoints where the flux tube arch crosses a gravitational equipotential surface, along which the total pressure is constant, then the total pressure inside the flux tube is the same at the two footpoints. An outward siphon flow in the flux tube will be driven when the gas pressure in the tube is lower at the outer footpoint than at the inner footpoint, which requires that the magnetic pressure in the tube be higher at the outer footpoint than at the inner footpoint. As first pointed out by Spruit (1981), this is precisely the situation for many penumbral flux tubes, for which the magnetic field strength at the inner footpoint is around $1000 \mathrm{G}$ (a typical penumbral field strength) but at the outer footpoint is of order $1500 \mathrm{G}$, corresponding to a typical intense, collapsed photospheric magnetic element.

Most of the magnetic flux in the quiet photosphere is concentrated into such highly evacuated flux tubes, which form naturally because of a convective collapse mechanism associated with the strongly superadiabatic temperature gradient in the thin upper boundary layer (the granulation layer) of the solar convection zone. Early work on the convective collapse mechanism treated it as an instability of a vertical flux tube at rest in a static superadiabatic atmosphere (see the review by Spruit \& Roberts 1983). However, in the granulation layer on the Sun, convective collapse and flux expulsion are different aspects of the same dynamical process (Hughes \& Proctor 1988; Schüssler 2001). Numerical simulations of compressible magnetoconvection show that strong, partially evacuated magnetic flux tubes are formed in the rapidly sinking plumes (Hurlburt \& Toomre 1988; Grossmann-Doerth et al. 1994; Grossmann-Doerth, Schüssler, \& Steiner 1998; Weiss et al. 1996; Steiner et al. 1996, 1998) merely by the action of the converging flows and the subsequent concentration of magnetic field as the fluid attempts to maintain total pressure equilibrium. Because the strong downward plumes are also responsible for the flux pumping, we now see that there is an intimate connection between flux pumping and convective collapse. Penumbral flux tubes that are pumped downward by the descending plumes in intergranular lanes will quite naturally collapse to an intense magnetic element at the outer footpoint, producing the drop in gas pressure that drives the Evershed outflow.

This outflow is known to be time dependent, with flows along individual flux tubes waxing and waning on a timescale of order 10-15 minutes (Shine et al. 1994; Rimmele 1994; Rouppe van der Voort 2003). This time dependence may be associated with variations in the gas pressure and magnetic field strength at the footpoints: at the outer footpoint as a result of the interaction with the granular convection (Thomas 1994), or at the inner footpoint as a result of variations associated with convection within the penumbra itself (Schlichenmaier et al. 1998a). Alternatively, the time dependence could be due to vertical excursions of flux tubes containing nearly steady flows (Rouppe van der Voort 2003). The outward-moving Evershed "clouds" seen in Dopplergrams of the penumbra (Shine et al. 1994) apparently originate from disturbances at the inner footpoints, although the fact that these features are coherent across several filaments suggests that they are associated with some larger scale wave motion rather than independent disturbances in individual flux tubes.

A variant of the siphon flow model has been proposed by Schlichenmaier (2002), in which cooling of a time-dependent flow along the flux tube is strong enough to produce a local density enhancement sufficient to render the tube negatively buoyant, causing it to sink locally and leading to a serpentine configuration for the flux tube. (The possibility of such a sinusoidal configuration of the flux tube was demonstrated earlier for steady state siphon flows by Thomas \& Montesinos 1990 , but they did not attach any particular significance to this configuration for the Evershed flow.) The serpentine configuration is associated with quite high flow velocities along the flux tube, however, so it is not clear whether this configuration actually occurs in a sunspot penumbra.

\section{MOVING MAGNETIC FEATURES AND THE MOAT}

Most sunspots are surrounded by an annular moat cell, 10-20 Mm wide, in which there is a persistent horizontal, radial outflow. The moat cell is essentially a large, annular supergranule, centered on the sunspot. The moat contains no permanent magnetic field, but small magnetic elements are observed to move radially outward across the moat with speeds ranging from a few tenths to $3 \mathrm{~km} \mathrm{~s}^{-1}$ (Sheeley 1969; Vrabec 1971; Harvey \& Harvey 1973; Zwaan 1992). These moving magnetic features (MMFs) have been classified into three types and their properties have been summarized by Shine $\&$ Title (2001); our discussion below closely follows their summary of the observations. Each of these types of MMFs can be interpreted physically in terms of the flux-pumping scenario, as shown schematically in Figure 13.

Type I MMFs consist of bipolar pairs of magnetic elements, with the inner element having the same polarity as the sunspot. The pairs of elements move outward together across the moat at speeds of $0.5-1 \mathrm{~km} \mathrm{~s}^{-1}$. They usually first appear just outside the sunspot along a radial line extending from a dark penumbral filament. These features can be interpreted quite naturally as the footpoints of magnetic loops that have 


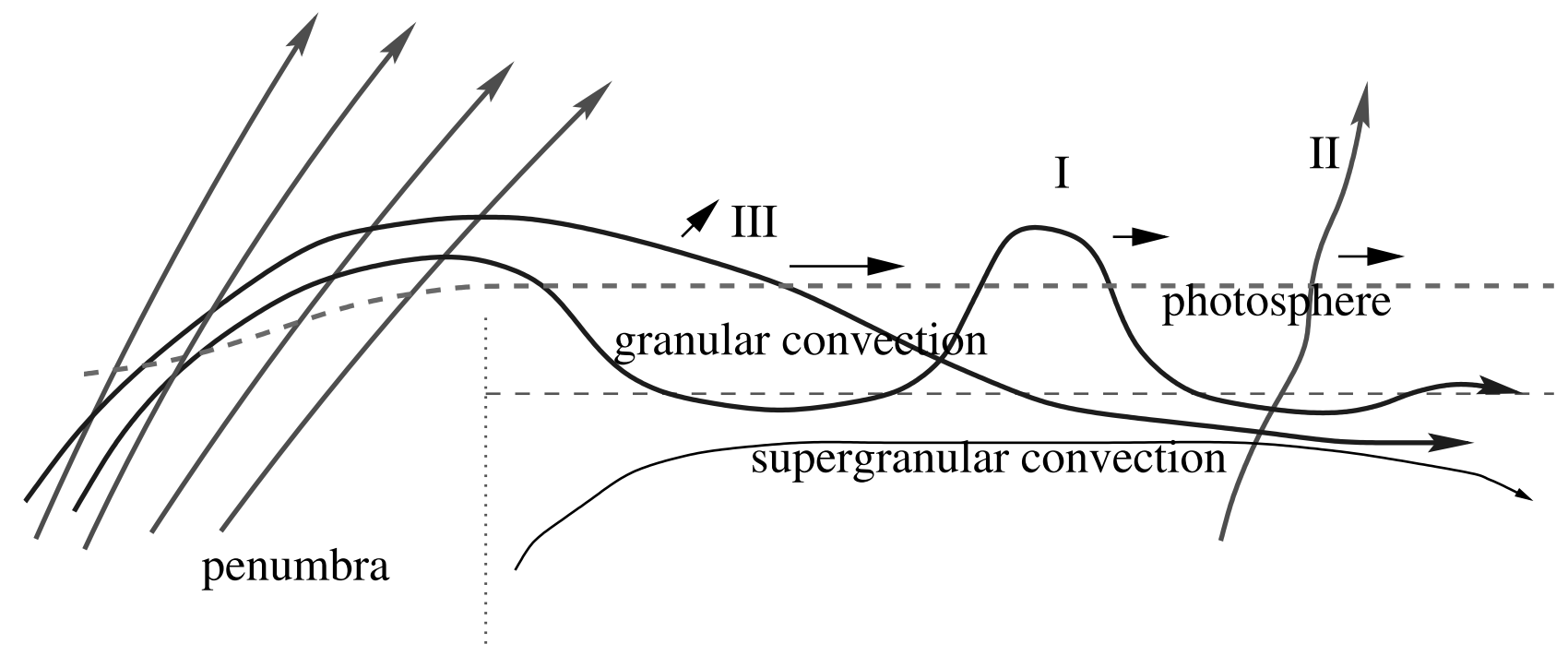

FIG. 13.-Sketch of MMFs in the moat around a sunspot (from Thomas et al. 2002b). In $\S 7$ the three types of MMFs (types I, II, and III) are interpreted in the context of magnetic flux pumping by granular convection in the moat. [See the electronic edition of the Journal for a color version of this figure.]

erupted through the solar surface at places along a submerged (downward-pumped) flux tube where there are particularly strong convective updrafts. These magnetic loops are then swept outward along with the granulation pattern by the moat flow, although the outward motion is often somewhat faster than the flow speed, indicating that the motion is at least partly due to the propagation of the loop as a kink wave along the flux tube. This picture is essentially the same as the model proposed by Harvey \& Harvey (1973; see their Fig. 6), except that here we give a physical explanation for the origin of the loops (called "twists" by Harvey \& Harvey 1973) that form along the flux tube. Several loops can emerge at different positions and at different times along the same flux tube, and this is reflected in the fact that successive type I MMFs tend to follow nearly identical paths across the moat. As long as the submerged flux tube remains attached to the sunspot, the type I MMFs that form along it are not associated with a decay of the sunspot.

Type II MMFs are single magnetic elements with the same polarity as the sunspot, moving outward across the moat at speeds similar to that of type I MMFs. These features may be interpreted as flux tubes that have separated from the flux bundle forming the sunspot and are being carried away by the moat flow. This process is the primary means by which a sunspot decays.

Type III MMFs are single magnetic elements with polarity opposite to that of the sunspot, moving rapidly outward at speeds of $2-3 \mathrm{~km} \mathrm{~s}^{-1}$. These features can be explained by noting that the flux pumping is an average effect and that the downward-pumping force on an individual, strong flux tube may occasionally be too weak (perhaps because of the particular alignment of the tube with the convective pattern) to prevent that tube from rising under magnetic buoyancy and curvature forces. In this case the tube will emerge through the surface at a shallow angle, producing a rapid outward horizontal motion of the footpoint in the moat. Even while a returning flux tube remains submerged, buffeting by the turbulent convection will cause it to bob up and down somewhat, producing the inward and outward excursions of the outer edges of the dark penumbra filaments that are seen in movies of sunspots (Title et al. 1993).
Long-term averages of the moat flow show that the radial outflow is modulated azimuthally, with a superimposed pattern of azimuthal motions. These take the form of radial rolls, with alternating radial rays (or spokes) of convergence and divergence extending across the moat (Shine \& Title 2001). Within this pattern, the various MMFs seem to be preferentially channeled along the rays where the flow converges, whose separation is an order of magnitude greater than that of the penumbral filaments. This modulation may have a purely hydrodynamical origin, for axisymmetric Boussinesq convection in a cylindrical domain is known to be unstable to nonaxisymmetric modes (Jones \& Moore 1979), although any such instability is likely to be enhanced by the presence of submerged magnetic flux (Tildesley 2003).

The moat cell is surrounded by an irregular ring of magnetic field, with the same sign as that emerging from the spot, that gives rise to plages in $\mathrm{Ca}$ II emission. We can estimate the depth of the horizontal magnetic channels below the dark filaments as follows. Assume that the magnetic flux that emerges horizontally from the main sunspot flux tube below the solar surface is held pumped down across the entire moat cell and then pokes upward through the surface in an annular plage region at the periphery of the moat cell. Then the total flux emerging horizontally from the spot is equal to that emerging through the annular plage region. Let us take the radius of the moat cell to be twice the penumbral radius and assume that there is a plage annulus $5 \mathrm{Mm}$ wide around the moat with an average field strength of $300 \mathrm{G}$ and that magnetic flux emerges horizontally through about $70 \%$ of the circumference of the spot (consistent with the inclinations of the fields in bright and dark filaments and of the mean field) with a field strength of $1000 \mathrm{G}$ just outside the spot. Then we obtain a channel depth of 5-6 Mm, which seems quite reasonable. (Of course, not all the emerging flux may break through the surface in the plage region, and some of the flux in the plage region may come from fields that have escaped from the spot and are no longer connected to it.)

\section{FORMATION OF THE FILAMENTARY STRUCTURE}

The development of an active region starts with the emergence of a fragmented flux tube into the photosphere 
(Zwaan 1992). Magnetic fields are initially confined to tiny flux elements, which accumulate between granules and mesogranules to form small pores, without penumbrae (Keppens \& Martínez Pillet 1996; Leka \& Skumanich 1998). Some of these pores may then coalesce to form a sunspot. A typical pore has a central field of 1500-2000 G, and the inclination of the field to the vertical increases to an average value of around $35^{\circ}$ at the edge of the pore. Since the pore has to be in overall magnetohydrostatic equilibrium with its surroundings and the ambient gas pressure increases rapidly with depth, the flux tube beneath the pore must fan out as it approaches the surface (as in Fig. 2a). Simple models indicate that the inclination of the field at the boundary of the pore must increase as the total flux increases, and they suggest that there is a critical value of this inclination at which the pore develops a penumbra and becomes a sunspot (Simon \& Weiss 1970; Rucklidge, Schmidt, \& Weiss 1995; Hurlburt \& Rucklidge 2000). Observations show that this transition is associated with hysteresis: the largest pores are bigger than the smallest spots (Bray \& Loughhead 1964; Rucklidge et al. 1995; Skumanich 1999).

If we consider a simple pore model, with the flux tube embedded in a stratified atmosphere, the field at the edge of the flux tube (the magnetopause) is concave toward the plasma. Such a configuration is liable to magnetically driven interchange (or fluting) instabilities. It can be shown, however, that in an adiabatically stratified atmosphere the flux tube is stabilized by magnetic buoyancy provided that the radial component of the field at the magnetopause decreases upward (Meyer, Schmidt, \& Weiss 1977). Of course, the solar atmosphere is superadiabatically stratified and therefore unstable to convection both inside and outside the pore. Indeed, theoretical models of the umbrae of pores and spots show that most of the energy transport is by convection, up to a level just below the visible surface (Maltby 1992). The conjecture, therefore, is that, as the flux contained in the pore and hence the inclination of the field at the magnetopause are progressively increased, the configuration becomes unstable to convectively driven filamentary perturbations. The nonlinear development of this instability can then lead to fluting at the boundary and, eventually, to the formation of a penumbra with its interlockingcomb magnetic field.

To verify this conjecture, it is necessary first to construct an appropriate equilibrium model and then to study its stability. Hurlburt \& Rucklidge (2000) investigated axisymmetric, compressible magnetoconvection numerically and obtained steady nonlinear solutions. Preliminary results indicate that this configuration gives way to nonaxisymmetric patterns, with strong azimuthal variations in the magnetic field (Hurlburt et al. 2000; Hurlburt \& Alexander 2003). Now the scale of filamentation in a sunspot is small compared with the spot radius and the cylindrical geometry should not therefore be essential. A clearer picture of the filamentary instability emerges from a highly idealized Boussinesq model in Cartesian geometry (Tildesley 2003). This calculation starts from a fully nonlinear, asymmetric, two-dimensional equilibrium state, with a magnetic field that fans out in a manner similar to that in a pore (Weiss 1981). This state is subjected to perturbations that are periodic in the transverse ("azimuthal") direction, and it is found that there is a range of transverse wavenumbers for which the original two-dimensional configuration is unstable. In the nonlinear domain this instability saturates after developing into a broad spokelike pattern (Tildesley \& Weiss 2004). It turns out that, in this geometry and in the absence of a magnetic field, finite-amplitude two-dimensional rolls can become unstable to purely hydrodynamic modes; however, this only occurs for a very restricted range of wavenumbers, and the growth rate of the instability is considerably increased by the magnetic field. These results demonstrate the existence of three-dimensional, convectively driven, filamentary instabilities of two-dimensional magnetoconvective equilibria. In due course it should be possible to develop this approach so as to produce a more realistic model of a sunspot.

In the recent high-resolution images obtained by the Swedish $1 \mathrm{~m}$ Solar Telescope (Scharmer et al. 2002) even the smallest pores are surrounded by a thin "skirt," i.e., an annular ring with delicate fine-scale striations. This suggests that the convectively driven instability sets in at a very early stage in the formation of a sunspot. The initial perturbation apparently has a high azimuthal wavenumber, and it saturates at a low level. We conjecture that, as the inclination of the field increases, modes with larger and larger azimuthal scales become unstable, and that this process continues until the fluting matches the scale of the ambient granulation. Flux pumping by turbulent granular convection then adds an extra ingredient to the nonlinear development of this filamentary instability, for depressed flux can then be dragged downward by the sinking gas in intergranular lanes. Then there will be an abrupt transition to a new configuration with a fully developed penumbra. Indeed, observations show that the dark filaments appear abruptly and extend outward between the granules (Zwaan 1992). Conversely, as a sunspot decays, pumping can still keep fields in the dark filaments submerged when the total flux is less than that at which the transition from pores to spots occurs. This hysteresis indicates that the instability is associated with a subcritical bifurcation, as proposed by Rucklidge et al. (1995). Thus, the formation and maintenance of the penumbra, as well as its intricate magnetic structure, may well depend on magnetic pumping.

Following the onset of the convectively driven filamentary instability, the more horizontal spokes of magnetic field are brought into greater contact with the granular convective layer in the surroundings and hence are subject to downward pumping by the turbulent granular convection. As suggested by our simulations, some fraction of this more horizontal magnetic field will be pumped downward, forming the returning magnetic flux tubes, while the remainder will either stay above the surface or rise buoyantly to the surface out of the granulation layer, to form the magnetic canopy. The relative amounts of magnetic flux in the returning flux tubes and in the canopy remain an open question, both observationally and theoretically.

\section{CONCLUSIONS}

Our aim has been to show that downward pumping of magnetic flux by turbulent convection provides a mechanism that explains the structure and properties of the filamentary penumbra. We have argued that the fields in bright and dark filaments must remain distinct and that the latter are dragged downward by small-scale granular convection in the region outside the spot. To support this claim, we have carried out idealized numerical simulations of the flux pumping that occurs in the granular convection outside the sunspot. These simulations, carried out for a range of parameters, establish that the flux pumping is indeed a robust effect. This mechanism also explains the bistability associated with the transition from a pore to a sunspot and clarifies the behavior of the Evershed flow 
and the moving magnetic features in the moat cell surrounding a spot. Although some details of penumbral magnetoconvection can be understood, there is still much that needs to be explained. Nevertheless, it is clear that the gross structure of a sunspot, with its umbra and penumbra, can only be explained as a consequence of the processes that lead to fine structure in the penumbra. Fortunately, we are now able to resolve that fine structure on the Sun.

So far, we have not attempted to describe the subphotospheric structure of a sunspot. The field lines that emerge at low inclinations to form bright filaments form part of a flux bundle that is known to extend downward for at least $10 \mathrm{Mm}$ and may even continue to the base of the convection zone. The inner ends of the field lines in dark filaments must join this flux bundle too, but the depth of these filaments cannot be determined. We presume that the channels that contain them extend to a level at which downward pumping becomes ineffectual. The discussion in $\S 4.3$ implies that this level corresponds to the base of the layer of granular and mesogranular convection, at a depth of only a few megameters, and this is consistent with the behavior of moving magnetic features in the moat. Thus, these channels can only contain a small fraction of the total magnetic flux in the sunspot. Moreover, they will be embedded in the steeper fields of the bright filaments and convective transport will therefore be less effective in them. None of this implies, however, that the sunspot has to be a tight cluster of isolated flux tubes. Rather, we expect that at depths greater than a few megameters the magnetic field is contained within a single coherent but inhomogeneous flux bundle.

It is clear that much work has to be done in order to clarify the nature of convection in the bright and dark filaments and in the underlying plasma. In the near future, higher resolution observations of velocities and magnetic fields will become available. Meanwhile, more elaborate numerical simulations will reveal the pattern of convection and the deeper structure of the fields. The sunspot penumbra may be an old problem, but it raises subtle issues and there are many opportunities for new research.

We thank Valentin Martínez Pillet, Alastair Rucklidge, Mike Tildesley, and Alan Title for helpful discussions of various aspects of this paper. J. H. T. is grateful to the Department of Applied Mathematics and Theoretical Physics, University of Cambridge, for hospitality during a 6 month visit when many of the ideas expressed in this paper took shape. This research was supported by the UK Particle Physics and Astrophysics Research Council (N. O. W. and J. H. T.), the Sun-Earth Connection Theory program of the US National Aeronautics and Space Administration (N. H. B. and S. M. T.), and the Nuffield Foundation (S. M. T.).
Beckers, J. M. 1968, Sol. Phys., 3, 258

Beckers, J. M., \& Schröter, E. H. 1969, Sol. Phys., 10, 384

Börner, P., \& Kneer, F. 1992, A\&A, 259, 307

Brandenburg, A., Jennings, R. L., Nordlund, A., Rieutord, M., Stein, R. F., \& Tuominen, I. 1996, J. Fluid Mech., 306, 325

Bray, J. R., \& Loughhead, R. E. 1964, Sunspots (London: Chapman and Hall)

Brummell, N. H., Clune, T. L., \& Toomre, J. 2002, ApJ, 570, 825

Cattaneo, F., Brummell, N. H., Toomre, J., Malagoli, A., \& Hurlburt, N. E. 1991, ApJ, 370, 282

Cattaneo, F., Lenz, D., \& Weiss, N. O. 2001, ApJ, 563, L91

Danielson, R. E. 1961, ApJ, 134, 289

Degenhardt, D., \& Wiehr, E. 1991, A\&A, 252, 821

del Toro Iniesta, J. C. 2001, in ASP Conf. Ser. 248, Magnetic Fields across the Hertzsprung-Russell Diagram, ed. G. Mathys, S. K. Solanki, \& D. T. Wickramasinghe (San Francisco: ASP), 35

del Toro Iniesta, J. C., Bellot Rubio, L. R., \& Collados, M. 2001, ApJ, 549, L139

Dorch, S. B. F., \& Nordlund, Å. 2001, A\&A, 365, 562

Drobyshevski, E. M., \& Yuferev, V. S. 1974, J. Fluid Mech., 65, 33

Duvall, T. L., \& Gizon, L. 2000, Sol. Phys., 192, 177

Grossmann-Doerth, U., Knölker, M., Schüssler, M., \& Solanki, S. K. 1994, A\&A, 285, 648

Grossmann-Doerth, U., Schüssler, M., \& Steiner, O. 1998, A\&A, 337, 928

Harvey, J., \& Harvey, K. 1973, Sol. Phys., 28, 61

Hirzberger, J., \& Kneer, F. 2001, A\&A, 378, 1078

Hughes, D. W., \& Proctor, M. R. E. 1988, Annu. Rev. Fluid Mech., 20, 187

Hurlburt, N., \& Alexander, D. 2003, Adv. Space Res., 14, 19

Hurlburt, N. E., Matthews, P. C., \& Proctor, M. R. E. 1996, ApJ, 457, 933

Hurlburt, N. E., Matthews, P. C., \& Rucklidge, A. M. 2000, Sol. Phys., 192, 109

Hurlburt, N. E., \& Rucklidge, A. M. 2000, MNRAS, 314, 793

Hurlburt, N. E., \& Toomre, J. 1988, ApJ, 327, 920

Hurlburt, N. E., Toomre, J., Massaguer, J. M., \& Zahn, J.-P. 1994, ApJ, 421,245

Jones, C. A., \& Moore, D. R. 1979, Geophys. Astrophys. Fluid Dyn., 11, 245

Julien, K., Knobloch, E., \& Tobias, S. M. 2000, J. Fluid Mech., 410, 285 2003, in Advances in Nonlinear Dynamos, ed. A. Ferriz-Mas \&

M. Núñez-Jimenez (London: Taylor \& Francis), 195

Keppens, R., \& Martínez Pillet, V. 1996, A\&A, 316, 229

\section{REFERENCES}

Kosovichev, A. G., Duvall, T. L., \& Scherrer, P. H. 2000, Sol. Phys., 192, 159

Krivtsov, A. M., Hofmann, A., Staude, J., Klvaňa, M., \& Bumba, V. 1998, A\&A, 335, 1077

Lawrence, J. K., Cadavid, A. C., \& Ruzmaikin, A. A. 2001, Sol. Phys., 202, 27

Leka, K. D., \& Skumanich, A. 1998, ApJ, 507, 454

Lites, B. W., Elmore, D. F., Seagraves, P., \& Skumanich, A. P. 1993, ApJ, 418,928

Lites, B. W., Thomas, J. H., Bogdan, T. J., \& Cally, P. S. 1998, ApJ, 497, 464

Maltby, P. 1992, in Sunspots: Theory and Observations, ed. J. H. Thomas \& N. O. Weiss (Dordrecht: Kluwer), 103

Martínez Pillet, V. 1997, in ASP Conf. Ser. 118, Advances in the Physics of Sunspots, ed. B. Schmieder, J. C. del Toro Iniesta, \& M. Vázquez (San Francisco: ASP), 212

Matthews, P. C., Hurlburt, N. E., Proctor, M. R. E., \& Brownjohn, D. P. 1992, J. Fluid Mech., 240, 559

Mestel, L. 1998, Stellar Magnetism (Oxford: Clarendon)

Meyer, F., \& Schmidt, H. U. 1968, Z. Ang. Math. Mech., 48, T218

Meyer, F., Schmidt, H. U., \& Weiss, N. O. 1977, MNRAS, 179, 741

Moffatt, H. K. 1983, Rep. Prog. Phys., 46, 621

Montesinos, B., \& Thomas, J. H. 1997, Nature, 390, 485

Muller, R. 1992, in Sunspots: Theory and Observations, ed. J. H. Thomas \& N. O. Weiss (Dordrecht: Kluwer), 175

Nordlund, Å., Brandenburg, A., Jennings, R. L., Rieutord, M., Ruokalainen, J., Stein, R. F., \& Tuominen, I. 1992, ApJ, 392, 647

Ossendrijver, M., Stix, M., Brandenburg, A., \& Rüdiger, G. 2002, A\&A, 394, 735

Parker, E. N. 1963, ApJ, 138, 552

. 1979, Cosmical Magnetic Fields: Their Origin and Activity (Oxford: Clarendon)

Rädler, K.-H. 1968, Z. Naturforsch., 23, 1851

Rimmele, T. R. 1994, A\&A, 290, 972

. 1995a, A\&A, 298, 260

. 1995b, ApJ, 445, 511

Robinson, F. J., Demarque, P., Li, L. H., Sofia, S., Kim, Y.-C., Chan, K.-L., \& Guenther, D. B. 2003, MNRAS, 340, 923

Rouppe van der Voort, L. H. M. 2003, A\&A, 397, 757

Rucklidge, A. M., Schmidt, H. U., \& Weiss, N. O. 1995, MNRAS, 273, 491

Rüedi, I., Solanki, S. K., \& Keller, C. U. 1999, A\&A, 348, L37

Sams, B. J., III, Golub, L., \& Weiss, N. O. 1992, ApJ, 399, 313 
Scharmer, G. B., Gudiksen, B. V., Kiselman, D., Löfdahl, M. G., \& Rouppe van der Voort, L. M. H. 2002, Nature, 420, 151

Schlichenmaier, R. 2002, Astron. Nachr., 323, 303

Schlichenmaier, R., Jahn, K., \& Schmidt, H. U. 1998a, A\&A, 337, 897 1998b, ApJ, 493, L121

Schlichenmaier, R., \& Schmidt, W. 2000, A\&A, 358, 1122

Schüssler, M. 2001, in ASP Conf. Ser. 236, Advanced Solar Polarimetry: Theory, Observation and Instrumentation, ed. M. Sigwarth (San Francisco: ASP), 343

Sheeley, N. R., Jr. 1969, Sol. Phys., 9, 347

Shine, R. A., Simon, G. W., \& Hurlburt, N. E. 2000, Sol. Phys., 193, 313

Shine, R. A., \& Title, A. M. 2001, in Encyclopedia of Astronomy and Astrophysics, ed. P. Murdin (London: Nature Publishing Group), 3209

Shine, R. A., Title, A. M., Tarbell, T. D., Smith, K., Frank, Z. A., \& Scharmer, G. 1994, ApJ, 430, 413

Simon, G. W., \& Weiss, N. O. 1970, Sol. Phys., 13, 85

Skumanich, A. 1992, in Sunspots: Theory and Observations, ed. J. H. Thomas \& N. O. Weiss (Dordrecht: Kluwer), 121 1999, ApJ, 512, 975

Sobotka, M., Brandt, P. N., \& Simon, G. W. 1999, A\&A, 348, 621

Sobotka, M., \& Sütterlin, P. 2001, A\&A, 380, 714 2003, A\&A Rev., 11, 153

Solanki, S. K., \& Montavon, C. A. P. 1993, A\&A, 275, 283

Solanki, S. K., Montavon, C. A. P., \& Livingston, W. 1994, A\&A, 283, 221

Spitzer, L., Jr. 1957, ApJ, 125, 525

Spruit, H. C. 1981, in The Physics of Sunspots, ed. L. E. Cram \& J. H. Thomas (Sunspot: Sacramento Peak Obs.), 359

Spruit, H. C., Nordlund, Å., \& Title, A. M. 1990, ARA\&A, 28, 263

Spruit, H. C., \& Roberts, B. 1983, Nature, 304, 401

Stanchfield, D. C. H., II, Thomas, J. H., \& Lites, B. W. 1997, ApJ, 477, 485

Stein, R. F., \& Nordlund, A.. 1989, ApJ, 342, L95 1998, ApJ, 499, 914

Steiner, O., Grossmann-Doerth, U., Knölker, M., \& Schüssler, M. 1996, Sol. Phys., 164, 223 1998, ApJ, 495, 468

Stix, M. 2002, The Sun (2d ed.; Berlin: Springer)
Tao, L., Proctor, M. R. E., \& Weiss, N. O. 1998, MNRAS, 300, 907

Thomas, J. H. 1988, ApJ, 333, 407 1994, in Solar Surface Magnetism, ed. R. J. Rutten \& C. J. Schrijver (Dordrecht: Kluwer), 219

Thomas, J. H., \& Montesinos, B. 1990, ApJ, 359, 550

Thomas, J. H., \& Weiss, N. O. 1992, in Sunspots: Theory and Observations, ed. J. H. Thomas \& N. O. Weiss (Dordrecht: Kluwer), 3

Thomas, J. H., Weiss, N. O., Tobias, S. M., \& Brummell, N. H. 2002a, Astron. Nachr., 323, 383

2002b, Nature, 420, 390

Tildesley, M. J. 2003, MNRAS, 338, 497

Tildesley, M. J., \& Weiss, N. O. 2004, MNRAS, submitted

Title, A. M., Frank, Z. A., Shine, R. A., Tarbell, T. D., Topka, K. P., Scharmer, G., \& Schmidt, W. 1993, ApJ, 403, 780

Tobias, S. M., Brummell, N. H., Clune, T. L., \& Toomre, J. 1998, ApJ, 502, $\mathrm{L} 177$

. 2001, ApJ, 549, 1183

Vrabec, D. 1971, in IAU Symp. 43, Solar Magnetic Fields, ed. R. Howard (Dordrecht: Reidel), 329

Weiss, N. O. 1966, Proc. R. Soc. London A, 293, 310

1981, J. Fluid Mech., 108, 273

2002, Astron. Nachr., 323, 371

2003, in IAU Symp. 210, Modelling of Stellar Atmospheres, ed. N. E. Piskunov, W. W. Weiss, \& D. F. Gray (San Francisco: ASP), in press

Weiss, N. O., Brownjohn, D. P., Matthews, P. C., \& Proctor, M. R. E. 1996, MNRAS, 283, 1153

Weiss, N. O., Proctor, M. R. E., \& Brownjohn, D. P. 2002, MNRAS, 337, 293

Westendorp Plaza, C., del Toro Iniesta, J. C., Ruiz Cobo, B., Martínez Pillet, V., Lites, B. W., \& Skumanich, A. 1997, Nature, 389, 47

Winebarger, A. R., DeLuca, E. E., \& Golub, L. 2001, ApJ, 553, L81

Winebarger, A. R., Warren, H., van Ballegooijen, A., DeLuca, E. E., \& Golub, L. 2002, ApJ, 567, L89

Zel'dovich, Ya. B. 1956, J. Exp. Theor. Phys., 31, 154 (English transl. in Soviet Phys._JETP Lett., 4, 460 [1957])

Zwaan, C. 1992, in Sunspots: Theory and Observations, ed. J. H. Thomas \& N. O. Weiss (Dordrecht: Kluwer), 75 